TITLE:

\title{
Electronic stress tensor description of chemical bonds using nonclassical bond order concept
}

$\operatorname{AUTHOR}(S):$

Szarek, Pawel; Sueda, Yutaka; Tachibana, Akitomo

\section{CITATION:}

Szarek, Pawel ...[et al]. Electronic stress tensor description of chemical bonds using nonclassical bond order concept. JOURNAL OF CHEMICAL PHYSICS 2008, 129(9): 094102.

\section{ISSUE DATE:}

2008-09-07

URL:

http://hdl.handle.net/2433/84600

\section{RIGHT:}

Copyright 2008 American Institute of Physics. This article may be downloaded for personal use only. Any other use requires prior permission of the author and the American Institute of Physics. 


\title{
Electronic stress tensor description of chemical bonds using nonclassical bond order concept
}

\author{
Paweł Szarek, Yutaka Sueda, and Akitomo Tachibana ${ }^{\text {a) }}$ \\ Department of Micro Engineering, Kyoto University, Kyoto 606-8501, Japan
}

(Received 30 April 2008; accepted 29 July 2008; published online 2 September 2008)

\begin{abstract}
The stress tensors are used widely for description of internal forces of matter. For some time it is also applied in quantum theory in studies of molecular properties in chemical systems. Electronic stress tensors measure effects caused by internal forces acting on electrons in molecules and particularly those between bonded atoms. Utilized here stress tensor originated bond orders express bond strengths in terms of these internal forces. The unique concept of energy density and electronic chemical potential based bond orders gives natural evaluation of interaction strength compared with classical definition, considering delocalized nature of electrons. In addition to other causes, the relation to electronic energy may be used to predict relative stabilities of geometrical isomers or even conformers. (C) 2008 American Institute of Physics. [DOI: 10.1063/1.2973634]
\end{abstract}

\section{INTRODUCTION}

Recently an examination of bond order gains much attention regarding studies of multiple bonded atoms (especially metals). One has in choice various definitions expressing classical bond order concept by quantum mechanical methods usually in terms of density $y^{1,2}$ and overlap matrices ${ }^{3}$ or natural resonance theory (NRT). ${ }^{4}$ The bond order concepts have been reviewed recently by Mayer. ${ }^{5}$ The original idea traces back to Lewis's shared electrons and assigning it to bonding, nonbonding, or antibonding orbitals. However, due to disagreement between the experimental and theoretical results with simple Lewis-type structures for multiply bonded metal atoms, ${ }^{6,7}$ the use of nonclassically oriented approaches is advised. ${ }^{8}$ The delocalized nature of electron wave functions smeared over molecular orbitals (MOs) makes the assignment of electron pairs to particular bond quite challenging. Besides, these pairs might feel different about different elements; thus a bonding pair between two atoms may not be equivalent to a pair between the other two. New formulation of bond order concept ${ }^{8}$ presented here refers to the electronic energy rather than to electrons themselves; thus it is more suitable for evaluation of bond strength. Bond order was introduced as an indicator of bond stability and essentially refers to bond strength relative to single bonds; thus new concept of bond order expressed in terms of electronic energy density is formally and naturally better to fulfill this role. Moreover these new indices allow one to have a closer look on redistribution of energy over the molecule and its partition between particular bonds.

\section{THEORY}

The regional density functional theory (RDFT) method $^{9-15}$ has been used for energy density calculations. The method allows assigning the electronic energy density associated with the electron density at discrete points of real

${ }^{a)}$ Electronic mail: akitomo@scl.kyoto-u.ac.jp. space. ${ }^{16-19}$ The sum of regional contributions gives global electronic energy $E$, and the integration over the whole space leads to total energy. The kinetic energy density $n_{T}(\vec{r})$ obtained from energy density partitioning scheme within RDFT (Refs. 9 and 20-23) divides space into the electronic drop $R_{D}$ and atmosphere $R_{A}$ regions separated by the interface $S .{ }^{10} \mathrm{In}$ the electronic drop region, where $n_{T}(\vec{r})>0$, the electron density is simply accumulated and the classical motion of electrons is allowed. Contrary, the electron density is dried up and the motion of electron is classically forbidden in the complementary region $\left(n_{T}(\vec{r})<0\right)$ of the electronic atmosphere. The boundary $S$ in between $R_{D}$ and $R_{A}$ encloses the molecular region of reactant atoms and molecules along the course of the chemical reaction coordinate. The kinetic energy density is defined as

$$
\begin{aligned}
& n_{T}(\vec{r})=-\frac{\hbar^{2}}{4 m} \sum_{i}^{\text {occ }} \nu_{i}\left[\psi_{i}^{*}(\vec{r}) \Delta \psi_{i}(\vec{r})+\Delta \psi_{i}^{*}(\vec{r}) \psi_{i}(\vec{r})\right], \\
& R_{D}: \quad n_{T}(\vec{r})>0, \\
& R_{A}: \quad n_{T}(\vec{r})<0, \\
& S: \quad n_{T}(\vec{r})=0,
\end{aligned}
$$

where $m$ is the mass of the electron, $\psi_{i}(\vec{r})$ is the natural orbital, and $\nu_{i}$ is the occupation number of $\psi_{i}(\vec{r})$. The kinetic energy density is not positive definite in contrast to conventional positive-semidefinite ${ }^{24-29}$ or positive-definite ${ }^{30}$ representation. The calculations ${ }^{20-23}$ have not emphasized which representation of kinetic energy density is appropriate. The expectation values integrated over the whole space are the same. ${ }^{20}$ However, densities are different and the relevant differences are crucial for the study of chemical systems. The $n_{T}(\vec{r})=0$ defines boundary within which the intramolecular electric field produced by the other electrons does not exceed that of bare nucleus; ${ }^{10}$ thus the boundary $S$ defines the turning point for electron. 
The stress tensors are used to describe the internal forces of matter. In molecules it characterizes internal distortion of electron density. The local stresses, represented as the force acting on a pair of electronic drop regions ${ }^{10,14}$ of reactants, have been applied to study the chemical reactivity. ${ }^{13}$ The electronic stress tensor is derived according to

$$
\begin{aligned}
\tau^{S k l}(r)= & \frac{\hbar^{2}}{4 m} \sum_{i}^{\text {occ }} \nu_{i}\left[\psi_{i}^{*}(\vec{r}) \frac{\partial^{2} \psi_{i}(\vec{r})}{\partial x^{k} \partial x^{l}}-\frac{\partial \psi_{i}^{*}(\vec{r})}{\partial x^{k}} \frac{\partial \psi_{i}(\vec{r})}{\partial x^{l}}\right. \\
& \left.+\frac{\partial^{2} \psi_{i}^{*}(\vec{r})}{\partial x^{k} \partial x^{l}} \psi_{i}(\vec{r})-\frac{\partial \psi_{i}^{*}(\vec{r})}{\partial x^{l}} \frac{\partial \psi_{i}(\vec{r})}{\partial x^{k}}\right],
\end{aligned}
$$

with indices $k, l=1,2,3$. The electronic stress tensor is second rank tensor given by $3 \times 3$ matrix [Eq. (4)] with components defined by Eq. (3).

$$
\begin{aligned}
\left\langle\hat{\tau}_{\alpha}^{S}(\vec{r})\right\rangle & =\left[\begin{array}{ccc}
\tau_{\alpha x x}^{S}(\vec{r}) & \tau_{\alpha x y}^{S}(\vec{r}) & \tau_{\alpha x z}^{S}(\vec{r}) \\
\tau_{\alpha y x}^{S}(\vec{r}) & \tau_{\alpha y y}^{S}(\vec{r}) & \tau_{\alpha y z}^{S}(\vec{r}) \\
\tau_{\alpha z x}^{S}(\vec{r}) & \tau_{\alpha z y}^{S}(\vec{r}) & \tau_{\alpha z z}^{S}(\vec{r})
\end{array}\right] \\
& \underset{\operatorname{diag}}{\rightarrow}\left[\begin{array}{ccc}
\tau_{\alpha}^{S 11}(\vec{r}) & 0 & 0 \\
0 & \tau_{\alpha}^{S 22}(\vec{r}) & 0 \\
0 & 0 & \tau_{\alpha}^{S 33}(\vec{r})
\end{array}\right], \\
\tau_{\alpha}^{S 11}(\vec{r}) & \leq \tau_{\alpha}^{S 22}(\vec{r}) \leq \tau_{\alpha}^{S 33}(\vec{r}) .
\end{aligned}
$$

The eigenvalues of the stress tensor compose a set of invariant quantities that are the principal stresses. Their direction vectors (eigenvectors) are the principal directions. For surfaces normal to principal axes, the stress corresponds to pushes (negative) or pulls (positive) perpendicular to the surfaces. The very low negative stress regions are associated with atomic core regions, where electron density is highly compressed. The covalent bond formation is characterized by a concept of spindle structure. ${ }^{14}$ It is anticipated when the region, where the principal electronic stress is positive (tensile stress), along the line of the principal axis is connected to a pair of the $R_{D}$ 's of atoms or molecules (with predominant compressive stress). The electronic energy is given by the first invariant of the stress tensor. The half of the trace over eigenvalues of stress tensor gives local contribution $\left(\varepsilon_{\tau}(\vec{r})\right)$ to potential energy density of electrons [Eq. (6)].

$$
\varepsilon_{\tau}^{S}(\vec{r})=\frac{1}{2} \sum_{k} \tau_{\alpha}^{S k k}(\vec{r}), \quad E=\int d^{3} \vec{r} \varepsilon_{\tau}^{S}(\vec{r})
$$

It was shown in Ref. 15 that from the viral theorem, the following equation is given:

$$
\begin{aligned}
E_{\text {rigged QED }} & =\int d^{3} \vec{r}\left\langle\hat{H}_{\text {rigged QED }}\right\rangle \\
& =\int d^{3} \vec{r}\left\langle m_{e} c^{2} \hat{\bar{\psi}}(\vec{r}) \hat{\psi}(\vec{r})-\sum_{a} \hat{T}_{a}(\vec{r})\right\rangle,
\end{aligned}
$$

$$
\begin{aligned}
E_{\text {nonrelativistic rigged QED }} & =\int d^{3} \vec{r}\left\langle\hat{H}_{\text {nonrelativistic rigged QED }}\right\rangle \\
& =-\int d^{3} \vec{r}\left\langle\sum_{a} \hat{T}_{a}(\vec{r})\right\rangle \\
& =-\frac{1}{2} \int d^{3} \vec{r}\left\langle\sum \hat{\tau}_{\alpha k}^{k k}(\vec{r})\right\rangle,
\end{aligned}
$$

thus the integral over the trace of stress tensor density is equal to two times the kinetic energy density. The performance of other stress tensors ${ }^{29,31-35}$ was not studied in the context of this work (but were used to derive electronic pressure) so we cannot advocate for any definition. However, the formulation of the stress tensor directly translates into body forces that might represent different effects associated with charge distribution in molecules.

The total electronic force density operator is given by

$$
\hat{\vec{F}}_{\alpha}^{S}(r)=\vec{\tau}_{\alpha}^{S}(\vec{r})+\hat{L}_{\alpha}^{S}(\vec{r}),
$$

where $\hat{L}_{\alpha}^{S}(\vec{r})$ is the Lorentz force density operator and $\hat{\tau}_{\alpha}^{S}(\vec{r})$ is the tension density operator. The Lorentz force density operator consists the classical form and quantum mechanical exchange effects. The tension density operator represents purely quantum mechanical effects. In the stationary state of charged particles the local force vanishes since the tension density (the tension of the field) exactly cancels the Lorentz force density (the Lorentz force exerted on the particle) at every point of space [Eq. (10)]. . $^{12,14,36}$

$$
\left\langle\hat{\tau}_{\alpha}^{S}(\vec{r})\right\rangle+\left\langle\hat{L}_{\alpha}^{S}(\vec{r})\right\rangle=0 .
$$

The Lorentz force density operator is given by

$$
\begin{aligned}
& \hat{\vec{L}}(\vec{r})=\overrightarrow{\vec{L}}_{e}(\vec{r})+\sum_{a} \overrightarrow{\vec{L}}_{a}(\vec{r}), \\
& \hat{L}_{e}(\vec{r})=\hat{E}(\vec{r}) \hat{\rho}_{e}(\vec{r})+\frac{1}{c} \hat{j}_{e}(\vec{r}) \times \vec{B}(\vec{r}), \\
& \hat{\vec{L}}_{a}(\vec{r})=\hat{\vec{E}}(\vec{r}) \hat{\rho}_{a}(\vec{r})+\frac{1}{c} \hat{j}_{a}(\vec{r}) \times \hat{B}(\vec{r}),
\end{aligned}
$$

where $\vec{L}_{e}(\vec{r})$ is the electronic Lorentz force density operator and $\hat{L}_{a}(\vec{r})$ is the Lorentz force density operator of atomic nucleus $s .{ }^{15}$ The theory should be valid for atoms and molecules and for harmonically bound electrons if the harmonic potential is a "model" of the underlying electromagnetic interaction. The tension is given as the divergence of the stress tensor density operator

$$
\begin{aligned}
\hat{\tau}_{\alpha}^{S k}(\vec{r})= & \partial_{l} \hat{\tau}_{\alpha}^{S k l}(\vec{r}) \\
= & \frac{\hbar^{2}}{4 m} \sum_{i}^{\text {occ }} \nu_{i}\left[\psi_{i}^{*}(\vec{r}) \frac{\partial \Delta \psi_{i}(\vec{r})}{\partial x^{k}}-\frac{\partial \psi_{i}^{*}(\vec{r})}{\partial x^{k}} \Delta \psi_{i}(\vec{r})\right. \\
& \left.+\frac{\partial \Delta \psi_{i}^{*}(\vec{r})}{\partial x^{k}} \psi_{i}(\vec{r})-\Delta \psi_{i}^{*}(\vec{r}) \frac{\partial \psi_{i}(\vec{r})}{\partial x^{k}}\right] .
\end{aligned}
$$

In the stationary state between chemically bonded atoms, 

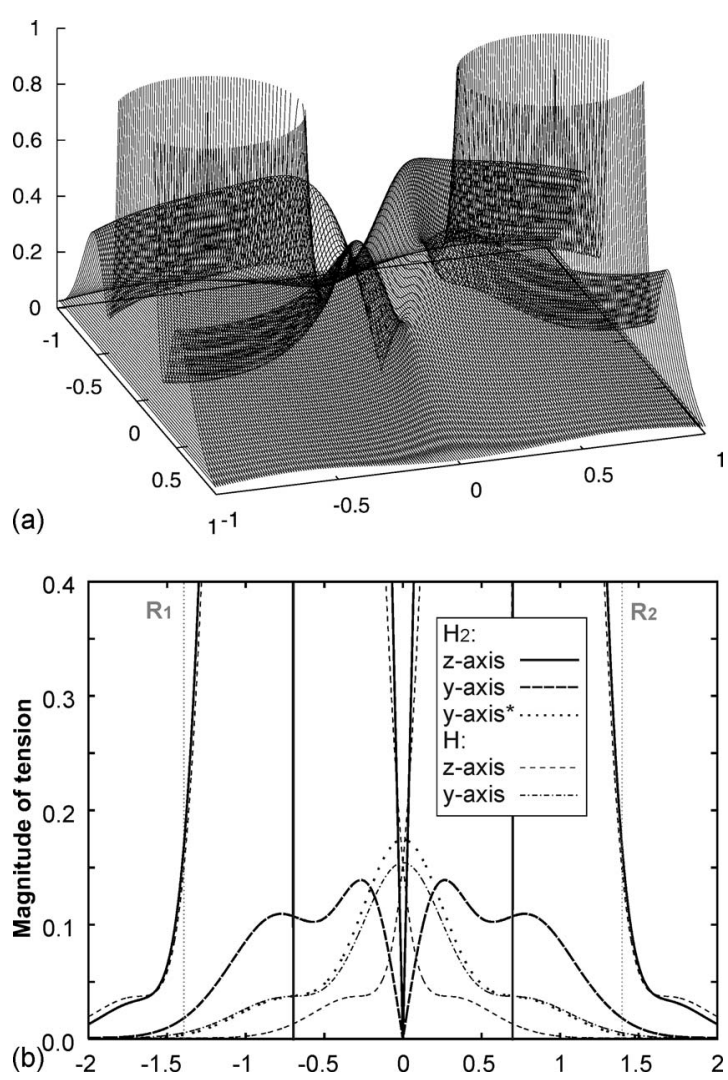

FIG. 1. Magnitudes of tension in $\mathrm{H}_{2}$ molecule compared with the values in isolated $\mathrm{H}$ atoms, distance in angstroms: (a) In the $\mathrm{H}_{2}$ molecular plane and (b) in the $\mathrm{H}_{2}$ molecule along the interatomic direction ( $z$-axis) and along perpendicular directions going through the Lagrange point at the center of symmetry ( $y$-axis) and through points $R_{1}$ and $R_{2}$ outside the interatomic region of $\mathrm{H}_{2}$ at distance of half of the $\mathrm{H}_{1}-\mathrm{H}_{2}$ bond length ( $y$-axis*). Also superimposed are the comparative values in a pair of isolated $\mathrm{H}$ atoms situated at $\mathrm{H}_{1}$ or $\mathrm{H}_{2}$ positions without interaction.

within electronic drop region, one can localize peculiar point (the Lagrange point $\vec{r}_{\text {Lagrange }}$ ) at which repulsive electronic tension (and also the Lorentz force) cancels itself in space; thus the local tension density is zero. ${ }^{8}$

\section{A. Calculation method}

The molecules were optimized at $\mathrm{HF} / 6-311++\mathrm{G}^{* *}$ level of theory using GAUSSIAN 03 program package ${ }^{37}$ (G03) unless specified different. The corresponding wave functions were used as input for RDFT code for electronic stress tensor and energy density calculations. ${ }^{38}$ The natural bond orbital (NBO) bond orders were calculated using G03 and Mayer's bond orders using APOST and BORDER programs. ${ }^{39,40}$

\section{B. The nature of the Lagrange point}

Figure 1(a) shows the absolute value of tension force density along the interatomic axis in $\mathrm{H}_{2}$ molecule and in hydrogen atom and Fig. 1(b) shows the tension density in the cross-section plane of $\mathrm{H}_{2}$. The origin corresponds to the Lagrange point $\left(r_{\text {Lagrange }}\right)$ of $\mathrm{H}_{2}$ molecule. The Lagrange point exists in the molecule, between bonded hydrogen atoms, where bond lines (eigenvectors of stress) form an envelope connecting atomic centers. ${ }^{8}$ It does not appear for single hydrogen atom or outside the interatomic region of hydrogen molecule. The greater the internal quantum forces are the faster the probability density changes in space are. At $r_{\text {Lagrange }}$ internal quantum forces disappear (for stationary state). The Lagrange point is an attractor for fluxes and is observed where the density builds up (it is observed also at the atomic nuclei position) and the interference pattern forms $\left(r_{\text {Lagrange }}\right.$ results from intense interference $)$. At the Lagrange point the net force is zero; thus this point does not produce any acceleration/deceleration of electrons. It also implies zero energy density difference (no electronic "pressure" change) or zero momentum transfer. This is the stationary point of the charge density in the interatomic region between bonded atoms.

The saddle point of the electron density distribution does not have to indicate the stationary point for electrons since force exerted on particles might be nonzero. The $r_{\text {Lagrange }}$ has a mechanical origin and is determined by dynamical forces acting on electrons in contrast to bond critical point (BCP) of the atoms-in-molecules (AIM) theory, ${ }^{29}$ which is a topological parameter. Sometimes these two points are equal (for bonds in very symmetric molecules), but it is not a rule. In particular cases, when the BCP exists, the Lagrange point was not found because "body" forces were not balanced (no stationary point), like between two hydrogen atoms in the bay region in phenanthrene. ${ }^{41}$

The RDFT Lagrange points in molecules are like Lagrange points in astrophysics (i.e., Trojan asteroids or Kordylewski clouds). However, instead of gravitation, they are born from electromagnetic forces between atomic nuclei and much lighter electrons. The electromagnetic fields of two nuclei and electrons combined with orbital angular momentum (corresponding to the centrifugal force in classical physics) are balanced at Lagrange points, allowing electron density to be stationary with respect to atomic nuclei. The reason for existence of Lagrange points in molecules is wave function interference and interelectron resonance. These features make Lagrange point a specific connector between two chemically bonded atoms. The molecular properties at this point provide a reliable description of the bond nature.

Recently introduced bond order indices related to electronic stress tensor ${ }^{8}$ are based on quantities calculated at $r_{\text {Lagrange, namely, electronic energy density and electronic }}$ chemical potential. ${ }^{8}$ The quantities for a particular bond in a molecule are normalized by the corresponding ones in $\mathrm{H}_{2}$ molecule (as model, single, two-electron bond between two protons), calculated at the same level of theory (including method and basis set). The hydrogen molecule was chosen because it has no core electrons, thus constituting the simplest chemical bond. The $b_{\varepsilon}$ is the energy density bond order:

$$
b_{\varepsilon}=\frac{\varepsilon_{\tau A B}^{S}\left(\vec{r}_{\text {Lagrange }}\right)}{\varepsilon_{\tau H H}^{S}\left(\vec{r}_{\text {Lagrange }}\right)},
$$

and $b_{\mu}$ is the electronic chemical potential bond order: 


$$
\begin{aligned}
b_{\mu} & =\frac{b_{\varepsilon}}{\left(n_{A B}\left(\vec{r}_{\text {Lagrange }}\right) / n_{H H}\left(\vec{r}_{\text {Lagrange }}\right)\right)} \\
& =\frac{\varepsilon_{\tau A B}^{S}\left(\vec{r}_{\text {Lagrange }}\right)}{n_{A B}\left(\vec{r}_{\text {Lagrange }}\right)} \cdot\left(\frac{\varepsilon_{\tau H H}^{S}\left(\vec{r}_{\text {Lagrange }}\right)}{n_{H H}\left(\vec{r}_{\text {Lagrange }}\right)}\right)^{-1},
\end{aligned}
$$

where $\varepsilon(\vec{r})$ to $n(\vec{r})$ ratio (of fractions of the total electronic energy and the total number of electrons) gives linear approximation to chemical potential ${ }^{8}$ (local version of the result of Parr $e t \mathrm{al}^{42}$ ) at given point in space according to Eqs. (17) and (18).

$$
\begin{aligned}
& E_{A B}=\int d^{3} \vec{r} \varepsilon_{\tau A B}^{S}(\vec{r}), \quad N_{A B}=\int d^{3} \vec{r} n_{A B}(\vec{r}), \\
& \mu_{R}=\left(\frac{\partial E_{R}}{\partial N_{R}}\right) \Leftrightarrow \frac{\varepsilon_{\tau A B}^{S}(\vec{r})}{n_{A B}(\vec{r})} .
\end{aligned}
$$

\section{Atomic stabilization}

The positiveness of the largest eigenvalue of stress is an indicator of the covalent interaction between atoms when the spindle structure is formed. ${ }^{14}$ The largest eigenvalue is the resultant of effects along the interatomic (principal) axis, while two minor eigenvalues measure effects through the perpendicular plane. The electron pair sharing causes the electron withdrawing effect along the interatomic axis, which results in a positive stress. However, a triple carbon-carbon bond in acetylene molecule, without doubt covalent, shows only negative eigenvalues in the interatomic region. Such feature might be attributed to atomic stabilization effect. The atomic cores are characterized by very low negative (compressive) stresses. At long interatomic distances, such as single covalent bond equilibrium distance, the separation of negative core regions is sufficient enough and perturbation in the bonding region is meaningless. As two atoms come closer the core regions may affect the bonding region in a significant manner (semiunited atom), finally covering up the positive stress coming from the covalent interaction, like it has place in the case of acetylene.

The two lower eigenvalues (degenerate for single and triple bonds and split for double and aromatic bonds) decrease in close to a linear manner from single to double to triple bond [Fig. 2(a)]. The two lower eigenvalues of the double bond are not degenerate due to the $\pi$ symmetry plane. The degeneracy of two minor eigenvalues of the electronic stress tensor shows similar information like the bond ellipticity defined in the AIM theory. ${ }^{29}$ Table I compares the largest eigenvalue of stress at the Lagrange point of single, double, and triple carbon-carbon bonds for a group of hydrocarbons. The largest eigenvalue would change linearly from about +0.06 in single bond to about +0.0 for triple bond, if there was no atomic stabilization, because there was no reason for which such significant deviation from the linear behavior could occur. However, the largest eigenvalue of stress is negative by about -0.033 , which accounts for atomic stabilization.

The orbitalwise analysis of stress in carbon-carbon single to triple bonds indicates that the third MO (first va-
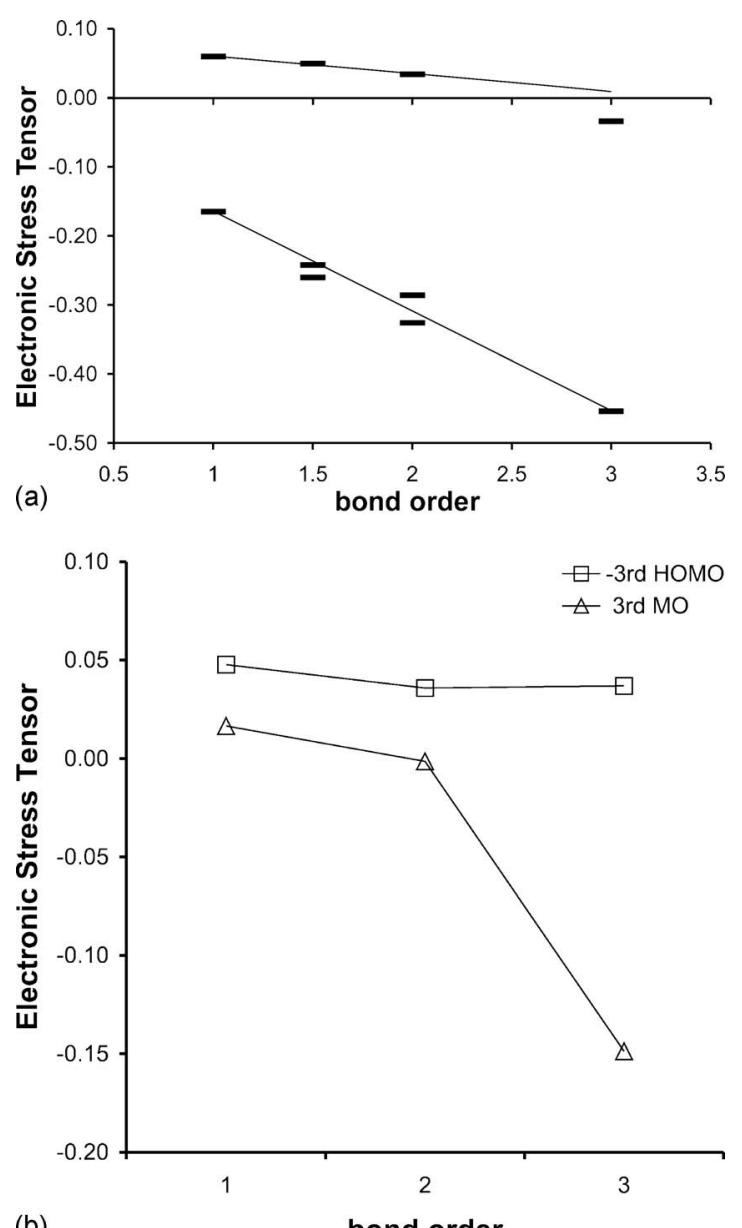

(b)

bond order

FIG. 2. The valence electrons MOs of ethane $(A 1-E 1)$, ethane $(A 2-E 2)$, and ethyne $(A 3-E 3)$. The $A$ is the third MO (first valence MO) and $E$ is HOMO. The $A$ and $C$ MOs give $70 \%-80 \%$ of total stress of carbon-carbon bond Lagrange point. The $E 3$ and $D 3$ MOs have the same symmetry and are perpendicular to each other.

lence $\mathrm{MO}$ ) and the third highest occupied molecular orbital (HOMO) are responsible for about $70 \%-80 \%$ of total electronic stress at the Lagrange point of carbon-carbon bond in $\mathrm{C}_{2} \mathrm{H}_{n=2,4,6}$ molecules. Moreover these two MOs do not

TABLE I. Largest eigenvalue of stress at the Lagrange point for single, double, and triple bonds in a group of hydrocarbons.

\begin{tabular}{lccc}
\hline \hline & Single & Double & Triple \\
\hline Ethane & 0.060 & & $\ldots$ \\
Ethene & $\ldots$ & 0.034 & $\ldots$ \\
Ethyne & $\ldots$ & $\ldots$ & -0.034 \\
Propane & 0.060 & $\ldots$ & $\ldots$ \\
Propene & 0.061 & 0.034 & $\ldots$ \\
Propyne & 0.059 & $\ldots$ & -0.033 \\
s-cis-butadiene & 0.060 & 0.035 & $\ldots$ \\
$s$-trans-butadiene & 0.059 & 0.035 & $\ldots$ \\
Vinylacetylene & 0.058 & 0.035 & -0.032 \\
1-butene & 0.060 & 0.034 & $\ldots$ \\
& 0.061 & $\ldots$ & $\ldots$ \\
1-butyne & 0.060 & $\ldots$ & -0.032 \\
& 0.059 & $\ldots$ & $\ldots$ \\
$n$-butane & 0.060 & $\ldots$ & $\ldots$ \\
Butadiyne & 0.054 & $\ldots$ & -0.033 \\
\hline \hline
\end{tabular}




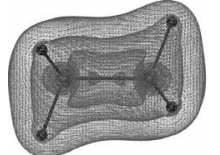

A 1

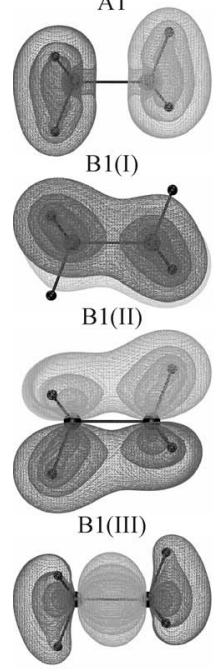

C3

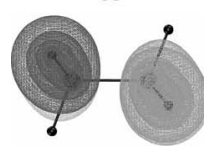

D1

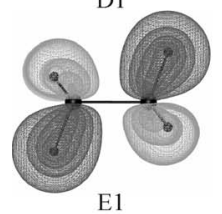

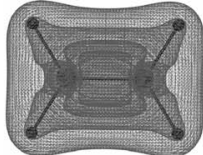

A2

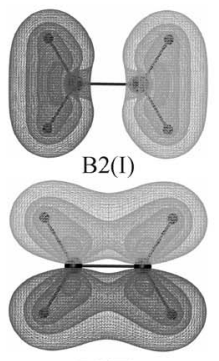

B2(II)

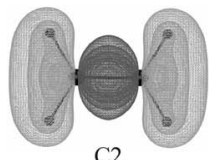

$\mathrm{C} 2$
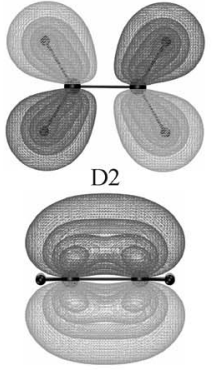

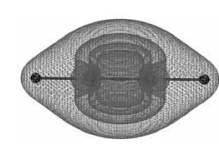

A3

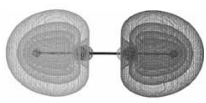

B3

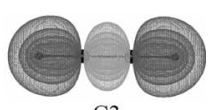

$\mathrm{C} 3$

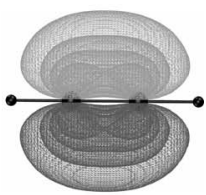

D3

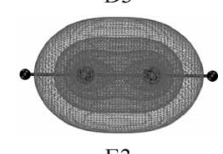

E3
FIG. 3. The eigenvalues of stress: (a) Change of eigenvalues of stress with bond order and (b) largest eigenvalue of stress for two valence MOs.

change the wave function phase (no nodal planes) in the interatomic region between carbon atoms. The MOs for valence electrons are shown in Fig. 3. The largest eigenvalues of stress of corresponding orbitals are compared in Fig. 2(b). The third HOMO eigenvalue shows an almost linear dependence with small deviation for double bond (due to $\pi$ symmetry), while the third MO eigenvalue significantly breaks linearity for triple bonds. The atomic stabilization, manifested by the negative stress of the triple covalent bond, might be attributed to low lying valence MOs, in the case of $\mathrm{C}_{2} \mathrm{H}_{n=2,4,6}$ molecules the first valence MO. Intuitively one might expect that low lying orbitals having strong likeness to core levels should be involved in such effects.

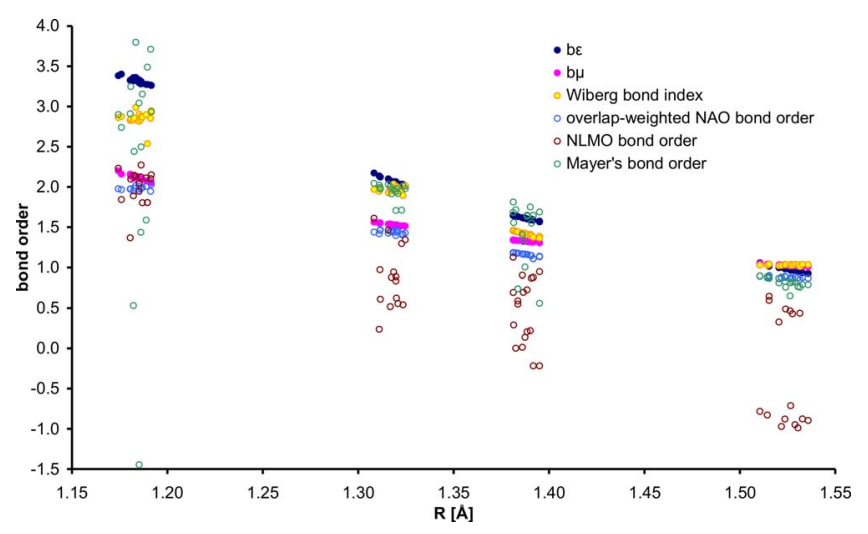

FIG. 4. (Color online) The correlation of carbon-carbon bond order indices with bond length for a group of organic compounds $\left(\mathrm{C}_{2} \mathrm{H}_{n} \mathrm{~A}\right.$ with different functional groups). The stress rooted bond orders $b_{\varepsilon}$ and $b_{\mu}$ are blue and pink dots; NBO bond orders: Wiberg's indices (yellow dot), atom-atom overlap NAO bond order (blue ring), and NLMO bond orders (brown ring), and Mayer's bond order (green ring).

\section{RESULTS AND DISCUSSION}

To illustrate how new bond order indices perform, a group of simple organic compounds with most common functional groups had been tested. The derivatives of ethane, ethane, and ethyne had been analyzed and the results were compared with Mayer's ${ }^{43}$ and NBO (Refs. 44-46) bond orders.

Figure 4 (see also Fig. S1 in supporting data ${ }^{47}$ ) shows a correlation of bond orders with bond length of $\mathrm{C}-\mathrm{C}$ bonds. The $b_{\varepsilon}$ and $b_{\mu}$ bond orders correlate very well with bond lengths for single, double, triple, and aromatic bonds while others have much worse coefficients (Table II). In general, when all bonds (single to triple) are considered together, the $b_{\varepsilon}$ and $b_{\mu}$ indices again show a very good correlation, with very similar coefficients, and the Wiberg index $\left(b_{W}\right)$ and atom-atom overlap natural atomic orbital (NAO) bond order $\left(b_{\mathrm{NAO}}\right)$ coefficients are alike. The Mayer's and natural localized molecular orbital (NLMO) $\left(b_{\mathrm{MO}}\right)$ indices do not correlate with bond length and assume negative values in certain cases. Some trends could be observed between energy density and NBO bond orders. The $b_{\varepsilon}$ coincides with the Wiberg index and $b_{\mu}$ is always higher than the atom-atom overlap NAO bond order values (Fig. 4). The stress tensor rooted indices are usually higher than the corresponding NBO bond orders.

Table III compares bond orders of several bonds from $\mathrm{C}_{2} \mathrm{H}_{5} \mathrm{~A}$ molecules. In most cases $b_{\mu}$ assume values similar or

TABLE II. The bond order vs bond length correlation coefficients of single, double, triple (of $\mathrm{C}_{2} \mathrm{H}_{n} \mathrm{~A}$, where $n=1,3,5$ and $\mathrm{A}$ are different functional groups), and aromatic (of phenyl group of $\mathrm{C}_{8} \mathrm{H}_{n=6,8,10}$ ) $\mathrm{C}$ to $\mathrm{C}$ bonds. Aberrations: $b_{\varepsilon}, b_{\mu}, b_{\mathrm{NAO}}, b_{\mathrm{NLMO}}, b_{M}$, and $b_{W}$ are the energy density, chemical potential, atom-atom overlapweighted NAO, natural localized orbital, Mayer's bond orders, and Wiberg's index, respectively.

\begin{tabular}{lcccrrr}
\hline \hline & $b_{\varepsilon}$ & $b_{\mu}$ & $b_{W}$ & $b_{\mathrm{NAO}}$ & $b_{\mathrm{NLMO}}$ & $b_{M}$ \\
\hline $\mathrm{C}-\mathrm{C}$ & -0.9884 & -0.9931 & -0.0099 & -0.6456 & -0.2049 & -0.6352 \\
$\mathrm{C}=\mathrm{C}$ & -0.9870 & -0.9916 & -0.0067 & -0.4478 & 0.0673 & -0.4172 \\
$\mathrm{C} \equiv \mathrm{C}$ & -0.9103 & -0.9699 & -0.1342 & -0.1040 & 0.1443 & -0.0238 \\
$\mathrm{C}-\mathrm{C}$ & -0.9913 & -0.9881 & -0.9660 & -0.8653 & -0.1635 & -0.0608 \\
In general & -0.9821 & -0.9823 & -0.9738 & -0.9820 & -0.8702 & -0.6569 \\
\hline
\end{tabular}


TABLE III. Stress tensor originated, NBO, and Mayer's bond orders of different bonds in $\mathrm{C}_{2} \mathrm{H}_{5} R$ molecules. The "av" subscript means arithmetical average over corresponding bonds of phenyl group. The number in superscript means successive hydrogen atom attached to a particular heavy atom. The $b_{\varepsilon}, b_{\mu}, b_{\mathrm{NAO}}, b_{\mathrm{NLMO}}, b_{M}$, and $b_{W}$ are the energy density, chemical potential, atom-atom overlap-weighted NAO, natural localized MO NLMO, Mayer's bond orders, and Wiberg's index, respectively.

\begin{tabular}{|c|c|c|c|c|c|c|c|}
\hline Bond & & $b_{\varepsilon}$ & $b_{\mu}$ & $b_{W}$ & $b_{\mathrm{NAO}}$ & $b_{\mathrm{NLMO}}$ & $b_{M}$ \\
\hline As $-\mathrm{H}^{1}$ & & 0.595 & 0.944 & 0.973 & 0.734 & 0.883 & 0.987 \\
\hline $\mathrm{As}-\mathrm{H}^{2}$ & & 0.597 & 0.944 & 0.975 & 0.734 & 1.732 & 1.003 \\
\hline C-As & & 0.442 & 0.805 & 0.930 & 0.744 & 0.092 & 1.075 \\
\hline $\mathrm{C}-\mathrm{Br}$ & & 0.457 & 0.818 & 0.993 & 0.721 & -1.124 & 0.846 \\
\hline $\mathrm{C}-\mathrm{C}$ & $\left(\mathrm{H}_{3} \mathrm{C}-\mathrm{CH}_{3}\right)$ & 0.964 & 1.020 & 1.039 & 0.873 & -0.712 & 0.815 \\
\hline $\mathrm{C}-\mathrm{C}_{(\mathrm{av})}$ & $\left(-\mathrm{C}_{6} \mathrm{H}_{5}\right)$ & 1.612 & 1.326 & 1.426 & 1.165 & 0.488 & 1.555 \\
\hline $\mathrm{C}-\mathrm{Cl}$ & & 0.636 & 0.922 & 0.992 & 0.729 & -1.091 & 0.903 \\
\hline $\mathrm{C}-\mathrm{F}$ & & 1.711 & 1.523 & 0.809 & 0.644 & 0.602 & 0.939 \\
\hline $\mathrm{C}-\mathrm{Ge}$ & & 0.457 & 0.853 & 0.846 & 0.749 & 0.231 & 1.018 \\
\hline $\mathrm{C}-\mathrm{H}$ & $\mathrm{C}_{2} \mathrm{H}_{6}$ & 1.180 & 1.106 & 0.958 & 0.809 & -0.029 & 0.994 \\
\hline $\mathrm{C}-\mathrm{H}_{\text {(av) }}$ & $\left(-\mathrm{C}_{6} \mathrm{H}_{5}\right)$ & 1.220 & 1.107 & 0.937 & 0.809 & 0.686 & 0.900 \\
\hline $\mathrm{C} \equiv \mathrm{N}$ & $(-\mathrm{CN})$ & 4.879 & 2.378 & 2.918 & 1.985 & 0.736 & 2.689 \\
\hline $\mathrm{C}-\mathrm{N}$ & $\left(\mathrm{C}_{2} \mathrm{H}_{5}-\mathrm{NH}_{2}\right)$ & 1.316 & 1.192 & 1.002 & 0.841 & 0.460 & 0.943 \\
\hline $\mathrm{C}-\mathrm{N}$ & $\left(\mathrm{C}_{2} \mathrm{H}_{5}-\mathrm{NO}_{2}\right)$ & 1.216 & 1.142 & 0.870 & 0.739 & -0.341 & 0.673 \\
\hline $\mathrm{C}=\mathrm{O}$ & $(-\mathrm{CHO})$ & 4.075 & 2.099 & 1.847 & 1.355 & 1.856 & 2.015 \\
\hline $\mathrm{C}=\mathrm{O}$ & $(-\mathrm{COOH})$ & 4.115 & 2.102 & 1.729 & 1.378 & 0.424 & 1.979 \\
\hline $\mathrm{C}-\mathrm{O}$ & $\left(\mathrm{C}_{2} \mathrm{H}_{5}-\mathrm{OH}\right)$ & 1.670 & 1.393 & 0.916 & 0.763 & 0.436 & 0.969 \\
\hline $\mathrm{C}-\mathrm{O}$ & $(-\mathrm{COOH})$ & 2.297 & 1.594 & 1.001 & 0.906 & 0.832 & 1.146 \\
\hline $\mathrm{C}-\mathrm{P}$ & & 0.566 & 0.850 & 0.948 & 0.769 & 0.324 & 0.521 \\
\hline $\mathrm{C}-\mathrm{S}$ & & 0.603 & 0.873 & 1.010 & 0.782 & -1.075 & 0.555 \\
\hline $\mathrm{C}-\mathrm{Se}$ & & 0.449 & 0.802 & 0.992 & 0.750 & 0.133 & 1.001 \\
\hline $\mathrm{C}-\mathrm{Si}$ & & 0.520 & 0.847 & 0.820 & 0.773 & 0.259 & 0.839 \\
\hline $\mathrm{Ge}-\mathrm{H}^{1}$ & & 0.536 & 0.950 & 0.936 & 0.769 & 0.778 & 0.952 \\
\hline $\mathrm{Ge}-\mathrm{H}^{2}$ & & 0.536 & 0.950 & 0.936 & 0.769 & -0.511 & 0.952 \\
\hline $\mathrm{Ge}-\mathrm{H}^{3}$ & & 0.537 & 0.950 & 0.939 & 0.769 & 1.616 & 0.961 \\
\hline $\mathrm{N}-\mathrm{H}$ & $\left(-\mathrm{NH}_{2}\right)$ & 1.843 & 1.346 & 0.876 & 0.755 & 0.557 & 0.972 \\
\hline $\mathrm{N}-\mathrm{O}^{1}$ & $\left(-\mathrm{NO}_{2}\right)$ & 4.516 & 2.107 & 1.543 & 1.176 & 0.822 & 1.783 \\
\hline $\mathrm{N}-\mathrm{O}^{2}$ & $\left(-\mathrm{NO}_{2}\right)$ & 4.533 & 2.109 & 1.556 & 1.179 & -1.002 & 1.875 \\
\hline $\mathrm{O}-\mathrm{H}$ & $(-\mathrm{OH})$ & 2.706 & 1.631 & 0.784 & 0.698 & 1.501 & 0.931 \\
\hline $\mathrm{O}-\mathrm{H}$ & $(-\mathrm{COOH})$ & 2.628 & 1.601 & 0.748 & 0.660 & 1.368 & 0.899 \\
\hline $\mathrm{P}-\mathrm{H}^{1}$ & & 0.712 & 0.953 & 0.977 & 0.740 & 0.810 & 0.986 \\
\hline $\mathrm{P}-\mathrm{H}^{2}$ & & 0.714 & 0.954 & 0.979 & 0.741 & 1.744 & 0.947 \\
\hline $\mathrm{Se}-\mathrm{H}$ & & 0.699 & 0.985 & 0.989 & 0.736 & 1.593 & 0.970 \\
\hline S-H & & 0.895 & 1.034 & 0.982 & 0.753 & 0.367 & 0.941 \\
\hline $\mathrm{Si}-\mathrm{H}^{1}$ & & 0.564 & 0.898 & 0.927 & 0.768 & 0.724 & 0.960 \\
\hline $\mathrm{Si}-\mathrm{H}^{2}$ & & 0.564 & 0.898 & 0.927 & 0.768 & -0.488 & 0.960 \\
\hline $\mathrm{Si}-\mathrm{H}^{3}$ & & 0.565 & 0.899 & 0.930 & 0.770 & 1.653 & 0.958 \\
\hline
\end{tabular}

very close to NBO or Mayer's bond orders and to classical values, indicating that the electron chemical potential of a particular bond is proportional to the electron density or overlap in the interatomic region. In few cases $(\mathrm{C}-\mathrm{F}, \mathrm{C}-\mathrm{O}$, $\mathrm{N}-\mathrm{H}, \mathrm{N}=\mathrm{O}$, and $\mathrm{O}-\mathrm{H})$ the $b_{\mu}$ index breaks the above trend. It suggests that these electron pairs have extra increased or decreased energy. This index is related to the electron chemical potential but also represents the relative energy per electron at the Lagrange point; thus the ratio of chemical potentials corresponds to the relative energy of the electron associated with a particular bond. The $b_{\varepsilon}$ values significantly differ from other bond orders in many cases. This is because bonding electrons, recognized as electron pairs by classically oriented bond orders, bind two atoms with different strengths, depending on the electronic energy. This index gives a measure of the corresponding electronic energy and bond strength. The average trend of the electron density might be described as increasing with decreasing energy. It means that the heavier the $b_{\varepsilon}$ index is the greater the associated electron density is; thus (in correspondence with density or overlap based bond orders) a stronger bond or in other words an energetically more favorable one is considered.

Figures S2 and S3 (supporting data ${ }^{47}$ ) plot new bond order indicators versus NBO and Mayer's bond orders of various bonds. Figure S2 $\left(b_{\varepsilon}\right.$ versus $\left.b_{\mu}\right)$ shows a decent resolution allowing to group bonds in classes (depending on bonding elements and effects of neighboring bonds, i.e., bonds in ethane derivatives have lower indices than ethane or ethyne). Figures S3(a) and S3(b) $\left(b_{\varepsilon}\right.$ or $b_{\mu}$ versus NBO and Mayer's) show a poor resolution for most bond types, allowing only for rough classification. Moreover Mayer's and NLMO indices seem to introduce a lot of "noise" with largely dispersed values. The $b_{\mu}$ makes better correlation 


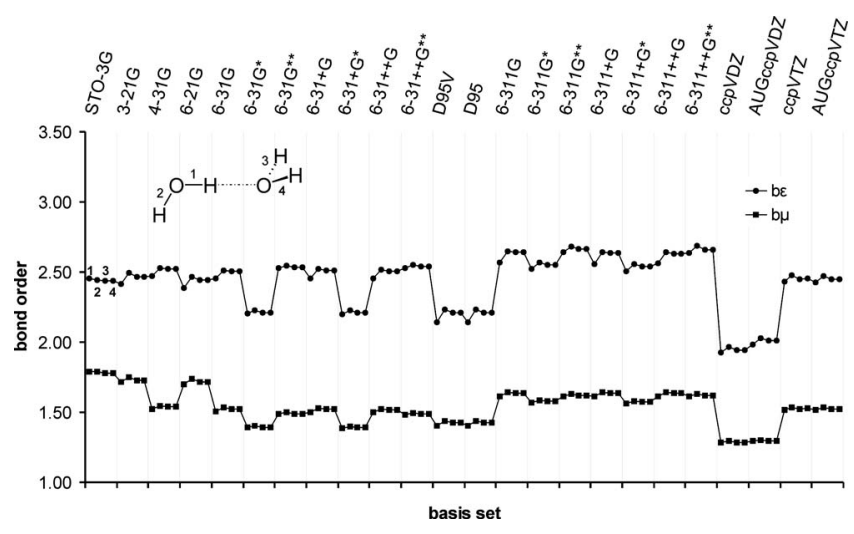

FIG. 5. Basis set dependence of $b_{\varepsilon}$ and $b_{\mu}$ bond orders of the O-H bonds for two water molecules connected by a hydrogen bond.

with all other indices than $b_{\varepsilon}$. Both indices correlate well with Wiberg and overlap-weighted NAO bond indices.

\section{A. Does basis set affect bond orders?}

The dependence of bond order indices against different basis sets implemented in GAUSSIAN (Ref. 37) had been tested. This comparison consists of STO-3G minimal basis set, $X-Y Z g$ and $X-Y Z W g$ various split valences, Pople basis sets, Dunning/Huzinaga valence (D95V), and full double-zeta $^{48}$ (D95) and Dunning's double (cc-pVDZ)- and triple-zeta (cc-pVTZ) correlation consistent basis sets. ${ }^{49,50}$ The tested systems as well as reference $\mathrm{H}_{2}$ molecules were optimized using a particular basis set.

The $b_{\varepsilon}$ index of covalent $\mathrm{O}-\mathrm{H}$ bonds in the $\left[\mathrm{H}_{2} \mathrm{O}\right]_{2}$ complex varies between 2.0 and 2.6 (Fig. 5); however, the range is much narrower $(2.35-2.5)$ for most basis sets and only Dunning/Huzinaga D95, D95V, and Dunning's correlation consistent double-zeta basis sets contribute to the lower limit of the index. The relevant bond order differences are in some part connected with different geometries obtained within particular basis set. One should note that these bond order indices correlate well with bond lengths and are sensitive to geometrical parameters of the system and in certain cases electronic properties, such as atomic charges or spin densities, predicted at a particular level of the theory. However, despite foregoing differences in bond orders, the particular trends regarding bond orders, i.e., of covalent $\mathrm{O}-\mathrm{H}$ bonds of hydrogen bond donor and acceptor water molecules, are well reproduced in all (except STO-3G) basis sets. Figures 5 and 6 show significant deviations of results for basis sets having polarization functions only on heavy atoms. The diffuse functions do not cause similar problems and its effect is hardly noticeable in this case. Similar trends and tendencies
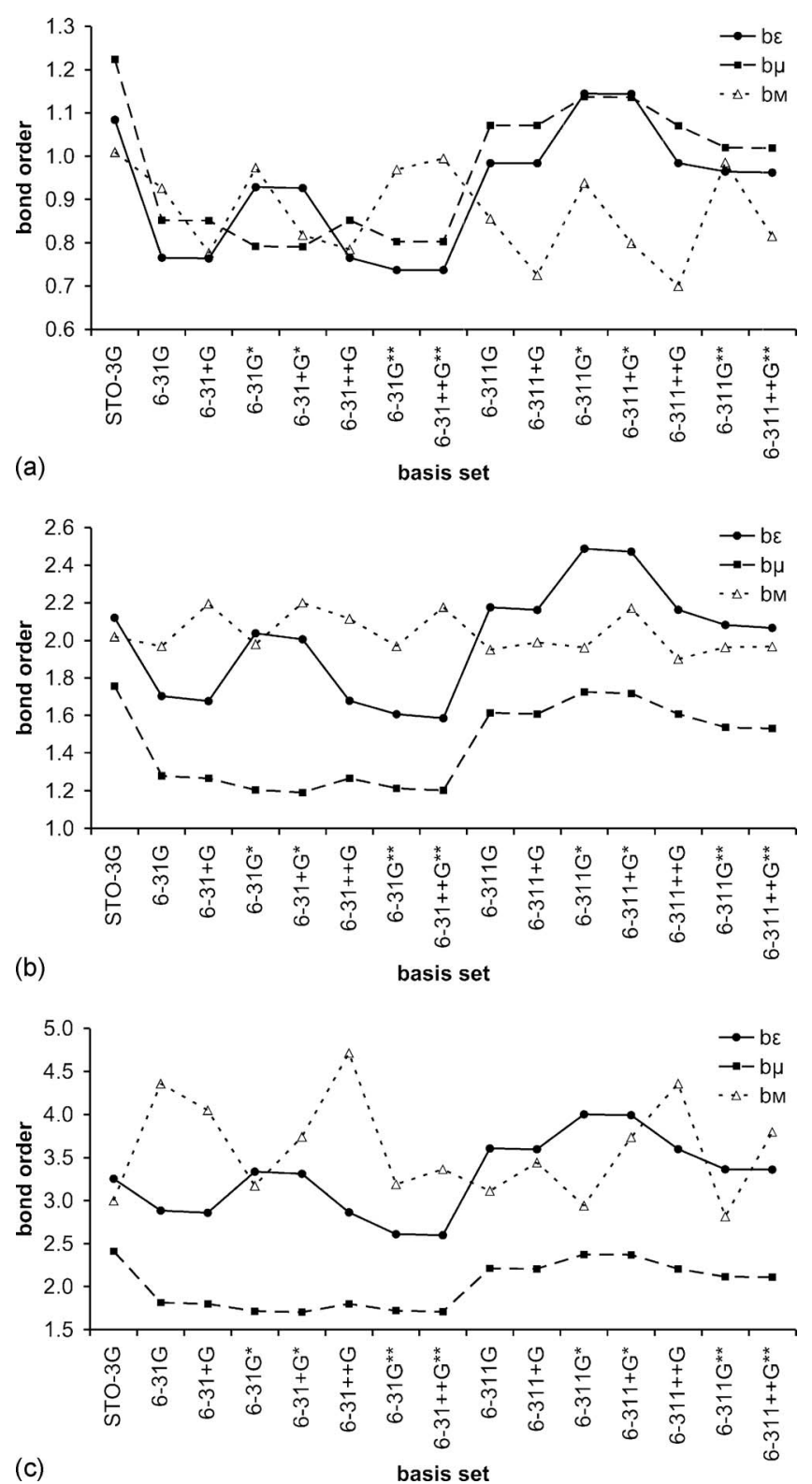

FIG. 6. Basis set dependence of $b_{\varepsilon}, b_{\mu}$, and Mayer's bond orders; the C to $\mathrm{C}$ bonds of $\mathrm{C}_{2} \mathrm{H}_{n=2,4,6}$ : (a) Single bond, (b) double bond, and (c) triple bond.

might also be observed for second-chemical potential weighted bond order index $b_{\mu}$; however, the values for corresponding bonds show much smaller dispersion.

The $b_{\varepsilon}, b_{\mu}$, and Mayer's indices show similar stability against different basis sets, as shown in Table IV. However, compared with Mayer's bond orders of carbon-carbon bonds in $\mathrm{C}_{2} \mathrm{H}_{n=2,4,6}$ molecules, the $b_{\varepsilon}$ (and also $b_{\mu}$ ) is advantageous, preserving the ratio of 1:2:3 for single, double, and triple

TABLE IV. Comparison of bond orders variation in STO-3G, 6-31G" , and 6-31G $\mathrm{G}^{\#}$ basis sets (number symbol denotes basis sets with diffuse and polarization functions added or removed).

\begin{tabular}{lcccccc}
\hline \hline & \multicolumn{3}{c}{ Average } & \multicolumn{3}{c}{ Maximum difference } \\
& $b_{M}$ & $b_{\varepsilon}$ & $b_{\mu}$ & $b_{M}$ & $b_{\varepsilon}$ & $b_{\mu}$ \\
\hline $\mathrm{C} \equiv \mathrm{C}$ & 3.843 & 4.358 & 2.785 & 1.544 & 1.422 & 0.708 \\
$\mathrm{C}=\mathrm{C}$ & 2.181 & 2.662 & 1.998 & 0.299 & 0.926 & 0.566 \\
$\mathrm{C}-\mathrm{C}$ & 0.933 & 1.229 & 1.330 & 0.310 & 0.423 & 0.432 \\
\hline \hline
\end{tabular}


bonds in all basis sets (Fig. 6), which turns out to be weakly conserved by Mayer's method especially in larger basis sets. ${ }^{51}$ The NBO bond orders show almost no dependency on applied basis set. The Wiberg index and NAO bond order show remarkable stability and preserve very similar values within all considered basis sets; the ratio of bond orders is also conserved.

Concluding, utilization of double-zeta basis sets results in lower bond orders. Although there are differences between indices obtained with different basis sets, the trends within particular basis set are preserved and the ratios of indices between different multiple bonds are conserved.

\section{B. Are $b_{\varepsilon}$ and $b_{\mu}$ reproducing horizontal and vertical trends in the Periodic Table?}

Figures $7(\mathrm{a})-7(\mathrm{c})$ show bond orders of $\mathrm{C}-\mathrm{A}$ bond in single substituted ethane, ethane, and ethyne derivatives, where A was chosen from $\mathrm{CH}_{3}, \mathrm{SiH}_{3}, \mathrm{GeH}_{3}, \mathrm{NH}_{2}, \mathrm{PH}_{2}$, $\mathrm{AsH}_{2}, \mathrm{OH}, \mathrm{SH}, \mathrm{SeH}, \mathrm{F}, \mathrm{Cl}$, and $\mathrm{Br}$. For C-A bonds the $b_{\varepsilon}$ index increases with group number and decreases with periods. A small exception is made by $\mathrm{GeH}_{3}$ group in ethylgermane of which the $\mathrm{C}-\mathrm{A} b_{\varepsilon}$ index is greater than that of bonds to elements from the following groups of the fourth period, placing its value near that of ethylsilane. The difference in bond orders of carbon bonds to elements of the same group but successive periods, namely, difference in $b_{\varepsilon}$ of second and third periods, is much greater than between third and fourth periods. The corresponding trend is stronger in the following groups. The $b_{\mu}$ index follows similar patterns, with exception that $b_{\mu}$ bond orders of $\mathrm{C}-\mathrm{Ge}$ bonds of germane species are higher than indices of $\mathrm{C}-\mathrm{Si}$ bonds in the corresponding silanes. This trend is more prominent among species with double and triple $\mathrm{C}$ to $\mathrm{C}$ bonds. This index reflects electronegativities of bounded partners (according to revised Pauling scale $\left.{ }^{52,53}\right)$. Such behavior is not surprising since electronegativity $\chi$ might be defined as the negative of the electronic chemical potential: ${ }^{42,54}$

$$
\chi=-\mu=-\left(\frac{\partial E}{\partial N}\right)_{v} .
$$

It was shown that bond orders express nature of bonded partners and $b_{\mu}$ is related to electronegativities.

\section{Conjugation and hyperconjugation effects in bond order representation}

Table V summarizes trends in bonding between carbon atoms when conjugation or hyperconjugation effects are present. One should notice that $b_{\varepsilon}$ bond orders of single bonds in $\mathrm{C}_{4} \mathrm{H}_{n}$ species, except the terminal single bond in butyne, are higher than in ethane molecule, while double and triple bond indices are lower than in ethene or ethyne molecules, respectively. Moreover bond orders of multiple bonds

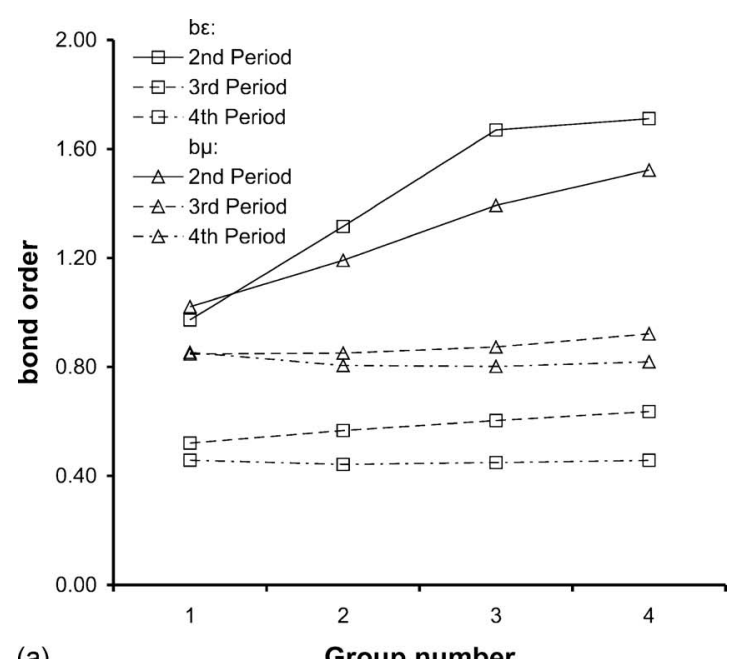

(a)

Group number

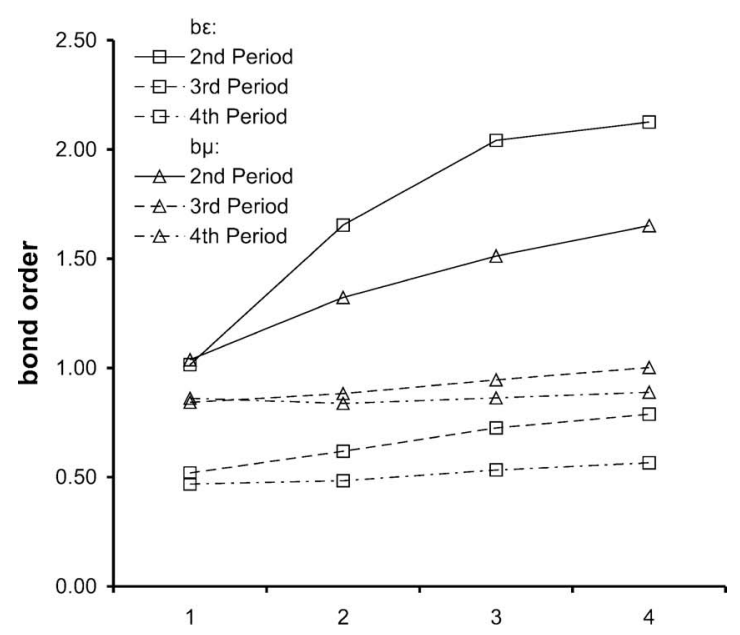

(b)

Group number

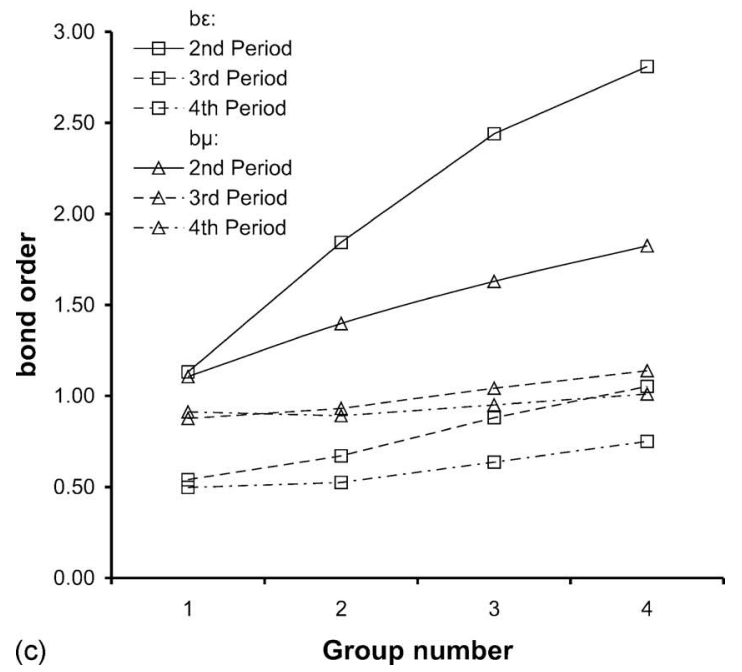

FIG. 7. The periodic trends of bond orders for $\mathrm{C}-\mathrm{A}$ bonds in $\mathrm{C}_{2} \mathrm{H}_{n} \mathrm{AH}_{m}$ (where $n=1,3,5$, and $\mathrm{A}$ is chosen from $\mathrm{C}$ to $\mathrm{F}$, $\mathrm{Si}$ to $\mathrm{Cl}$, and $\mathrm{Ge}$ to $\mathrm{Br}$ atoms, with $m=3,2,1,0$, respectively): (a) Ethane substitutions, (b) ethene substitutions, and (c) ethyne substitutions.

in butadiene, butadiyne, and vinylacetylene are also lower than the values of the corresponding bonds in butene or butyne. In the series of hydrocarbons the predicted strength of carbon-carbon bonds (Table V) indicated by $b_{\varepsilon}$ decreases in the following order. 
TABLE V. The stress tensor rooted bond orders $b_{\varepsilon}, b_{\mu}$, and Mayer's bond order $b_{M}$ of $\mathrm{C}-\mathrm{C}$ bonds of $\mathrm{C}_{2} \mathrm{H}_{m}$ and $\mathrm{C}_{4} \mathrm{H}_{n}$ species $(m=2,4,6$ and $n$ $=2,4,6,8,10)$.

\begin{tabular}{|c|c|c|c|c|c|c|c|c|c|}
\hline Molecule & $\mathrm{C} 1-\mathrm{C} 2$ & $\begin{array}{c}b_{\varepsilon} \\
\mathrm{C} 2-\mathrm{C} 3\end{array}$ & $\mathrm{C} 3-\mathrm{C} 4$ & $\mathrm{C} 1-\mathrm{C} 2$ & $\begin{array}{c}b_{\mu} \\
\mathrm{C} 2-\mathrm{C} 3\end{array}$ & $\mathrm{C} 3-\mathrm{C} 4$ & $\mathrm{C} 1-\mathrm{C} 2$ & $\begin{array}{c}b_{M} \\
\mathrm{C} 2-\mathrm{C} 3\end{array}$ & $\mathrm{C} 3-\mathrm{C} 4$ \\
\hline $\mathrm{CH}_{3}-\mathrm{CH}_{3}$ & 0.962 & $\cdots$ & $\cdots$ & 1.020 & $\cdots$ & $\cdots$ & 0.815 & $\cdots$ & $\cdots$ \\
\hline $\mathrm{CH}_{2}=\mathrm{CH}_{2}$ & 2.066 & $\cdots$ & $\cdots$ & 1.530 & $\cdots$ & $\cdots$ & 1.968 & $\cdots$ & $\cdots$ \\
\hline $\mathrm{HC} \equiv \mathrm{CH}$ & 3.362 & $\cdots$ & $\cdots$ & 2.110 & $\cdots$ & $\cdots$ & 3.798 & $\cdots$ & $\cdots$ \\
\hline $\mathrm{CH}_{2}=\mathrm{C}=\mathrm{CH}_{2}$ & 2.203 & 2.203 & $\cdots$ & 1.608 & 1.608 & $\cdots$ & 1.805 & 1.805 & $\cdots$ \\
\hline $\mathrm{CH}_{3}-\mathrm{CH}_{2}-\mathrm{CH}_{2}-\mathrm{CH}_{3}$ & 0.965 & 0.967 & 0.965 & 1.018 & 1.016 & 1.018 & 0.714 & 0.892 & 0.714 \\
\hline $\mathrm{CH}_{3}-\mathrm{CH}_{2}-\mathrm{CH}=\mathrm{CH}_{2}$ & 0.971 & 1.015 & 2.065 & 1.020 & 1.035 & 1.530 & 0.739 & 0.716 & 1.854 \\
\hline $\mathrm{CH}_{3}-\mathrm{CH}_{2}-\mathrm{C} \equiv \mathrm{CH}$ & 0.953 & 1.127 & 3.321 & 1.013 & 1.101 & 2.105 & 0.718 & 1.396 & -1.168 \\
\hline $\begin{array}{l}\mathrm{CH}_{2}=\mathrm{CH}-\mathrm{CH}=\mathrm{CH}_{2} \\
(s-c i s)\end{array}$ & 2.036 & 1.129 & 2.036 & 1.517 & 1.089 & 1.517 & 2.000 & 1.104 & 2.000 \\
\hline $\begin{array}{l}\mathrm{CH}_{2}=\mathrm{CH}-\mathrm{CH}=\mathrm{CH}_{2} \\
(\text { s-trans })\end{array}$ & 2.036 & 1.188 & 2.036 & 1.515 & 1.116 & 1.515 & 1.744 & 1.148 & 1.744 \\
\hline $\mathrm{CH}_{2}=\mathrm{CH}-\mathrm{C} \equiv \mathrm{CH}$ & 2.044 & 1.254 & 3.311 & 1.520 & 1.156 & 2.098 & 1.493 & 1.627 & -1.644 \\
\hline $\mathrm{HC} \equiv \mathrm{C}-\mathrm{C} \equiv \mathrm{CH}$ & 3.317 & 1.456 & 3.317 & 2.099 & 1.255 & 2.100 & 2.837 & 1.526 & 2.837 \\
\hline
\end{tabular}

(a) For triple bonds,

ethyne $>1$-butyne $>$ butadiyne $>$ vinylacetylene.

(b) For double bonds,

allene $>$ ethene $>1$-butene $>$ vinylacetylene

$$
>1,3 \text {-butadiene. }
$$

(c) For single bonds (middle),

$$
\begin{aligned}
\text { butadiyne } & >\text { vinylacetylene }>1,3 \text {-butadiene } \\
& >1 \text {-butyne }>1 \text {-butene }>n \text {-butane } \\
& >\text { ethane }>\text { twisted butane. }
\end{aligned}
$$

Furthermore the carbon-carbon single bond in $s$-trans-1,3-butadiene is stronger than in $s$-cis isomer. The above series indicates that conjugative $\pi_{a} \rightarrow \pi_{b}^{*}$ or hyperconjugative $\pi_{a} \rightarrow \sigma_{b}^{*}$ donor-acceptor interactions lead to partial bond equalization strengthening adjacent to the single bond and weakening double and triple bonds, which is consistent with resonance picture and experiment. Although the $b_{\varepsilon}$ index (Table V) shows no significant difference in strengths of double bonds of 1,3-butadiene isomers, the $b_{\mu}$ index (Table $\mathrm{V})$ of the $s$-cis isomer is higher than that of the $s$-trans. This behavior is consistent with natural resonance theory (NRT) bond order trend for these molecules, which indicates 1.939 and 1.950 for double bonds in s-trans and s-cis species, respectively. ${ }^{55}$ Additionally associated conjugative stabilization (s-trans of $-15.0 \mathrm{kcal} / \mathrm{mol}$ versus $s$-cis of $-12.8 \mathrm{kcal} / \mathrm{mol}$ ) (Ref. 55) in these molecules correlates with single carbon-carbon bond strength indicated by $b_{\varepsilon}$ bond order. The $b_{\varepsilon}, b_{\mu}$ (Table V), and NRT bond orders also show a good correlation for allene molecule (with NRT bond order of $\mathrm{C}=\mathrm{C}: 1.991) .{ }^{55}$ Greater bond orders of double bonds in allene than in 1,3-butadiene molecules do not indicate greater stability of the former molecule, which is the matter of reactivity dependent on many factors besides electronic energy and comes from interatomic contributions of all bonds in molecule, due to the delocalized nature of electrons, as well as from atomic core regions. Table VI shows sums of $b_{\varepsilon}$ indices of 1,3-butadiene isomers, allene, and propylene molecules. The "total" bond order is greater in more stable species. This is true for all bonds taken into account, likewise if only carbon-carbon bonds are summed, indicating the carbon chain as the main source of stability.

The energy density and chemical potential derived bond orders are able to distinguish relative stability of different geometrical isomers or even conformers. For instance, using $b_{\mu}$ index one can tell which of butane conformers is more stable. The $\Delta b_{\mu}$ for conformational change [Eq. (23)] is found to be -0.007 , which implies that the twisted butane molecule is less stable. The $\Delta b_{\mu}$ might be projected to enthalpy change (for reactions and processes with relatively small entropy contribution); thus interchange into "twisted" state is predicted to be slightly endothermic.

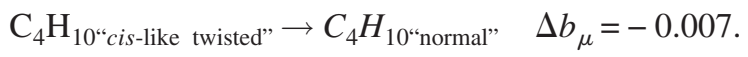

To estimate conjugation in diene and diyne we applied Kistiakowsky $^{56-59}$ method evaluating poliene stability by stepwise hydrogenation. The numbers over the arrows in re-

TABLE VI. The total $b_{\varepsilon}$ bond order of hydrocarbon isomers.

\begin{tabular}{lcc}
\hline \hline Molecule & $\begin{array}{c}\Sigma b_{\varepsilon} \\
\text { (all bonds) }\end{array}$ & $\begin{array}{c}\Sigma b_{\varepsilon} \\
(\mathrm{C}-\mathrm{C} \text { bonds })\end{array}$ \\
\hline$s$-cis-1,3-butadiene & 12.490 & 5.201 \\
s-trans-1,3-butadiene & 12.515 & 5.260 \\
Allene & 9.188 & 4.407 \\
Propyne & 9.283 & 4.462 \\
\hline
\end{tabular}



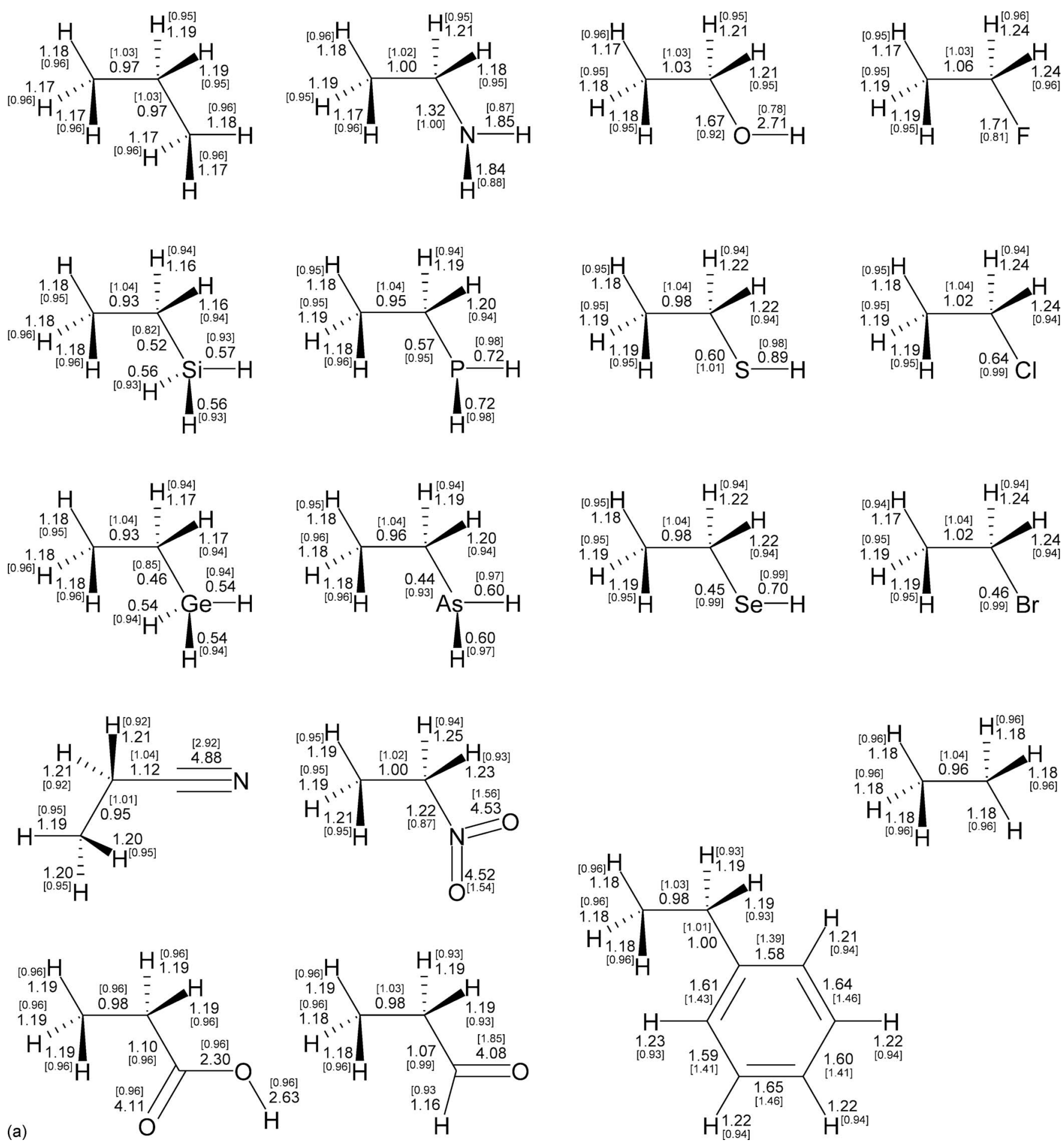

(a)
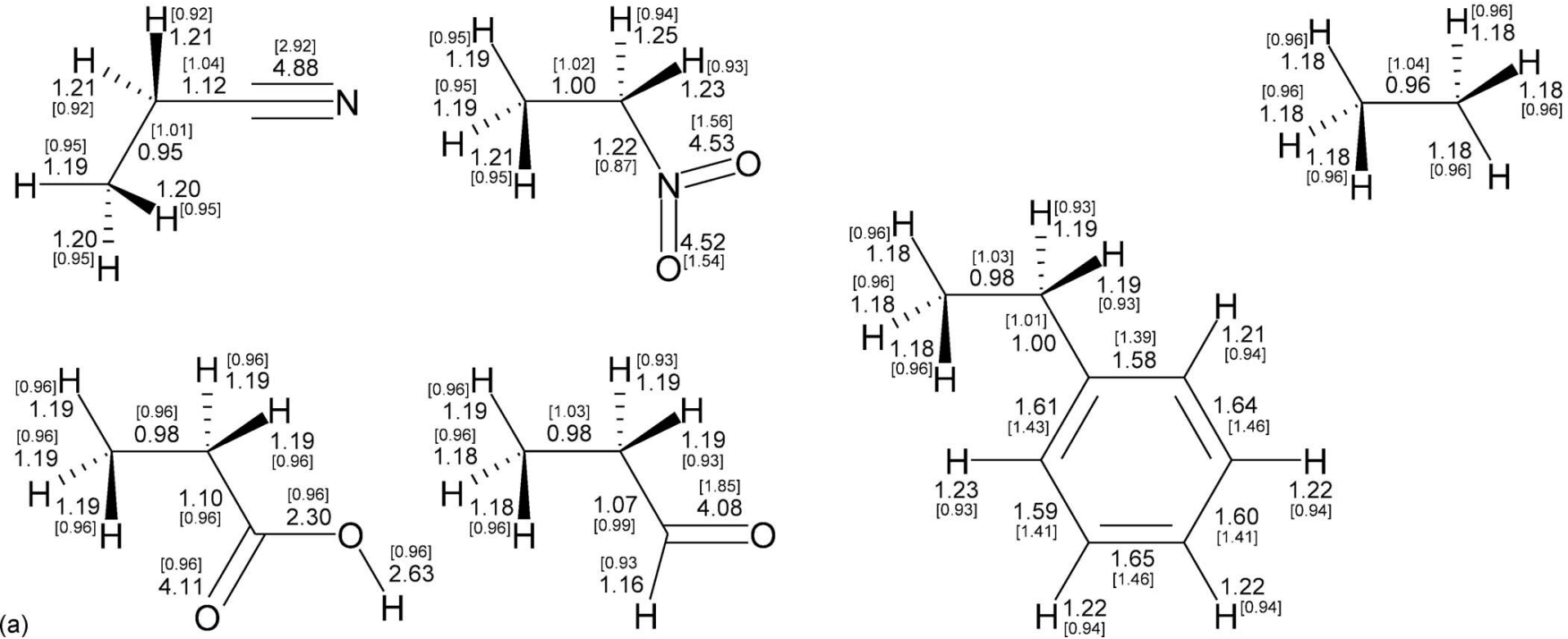

FIG. 8. The electronic stress tensor rooted bond orders of bonds in $\mathrm{C}_{2} \mathrm{H}_{5} \mathrm{~A}$ molecules $\left(\mathrm{A}\right.$ is $\mathrm{H}, \mathrm{CH}_{3}, \mathrm{CHO}, \mathrm{COOH}, \mathrm{CN}_{2} \mathrm{C}_{6} \mathrm{H}_{5}, \mathrm{SiH}_{3}, \mathrm{GeH}_{3}, \mathrm{NH}_{2}, \mathrm{NO}_{2}, \mathrm{PH}_{2}$, $\mathrm{AsH}_{2}, \mathrm{OH}, \mathrm{SH}, \mathrm{SeH}, \mathrm{F}, \mathrm{Cl}$, and $\mathrm{Br}$ ): (a) Energy density based bond order $b_{\varepsilon}$, Wiberg index in parentheses and (b) electronic chemical potential based bond order $b_{\mu}$, overlap-weighted NAO bond order in parentheses.

action equations indicate a difference between $b_{\mu}$ bond orders of all bonds being broken (reactants) and bond orders of bonds being formed (products).

$$
\begin{aligned}
s \text {-trans-1,3-butadiene }+ & \mathrm{H}_{2} \\
& -0.656 \\
& -0.688 \\
& \rightarrow \text { butane }
\end{aligned}
$$

$$
\text { 1,3-butadiyne }+2 \mathrm{H}_{2} \stackrel{-1.182}{\rightarrow} \text { 1-butyne }+2 \mathrm{H}_{2} \rightarrow-1.236 \text { butane }
$$

According to the difference of $\Delta b_{\mu}$ of first and second hydrogenation steps, the conjugation stabilization should be proportional to 0.032 and 0.054 (or -0.096 if corrected for atomic stabilization) for diene and diyne molecules, respectively. As can be seen atomic stabilization accounts for 

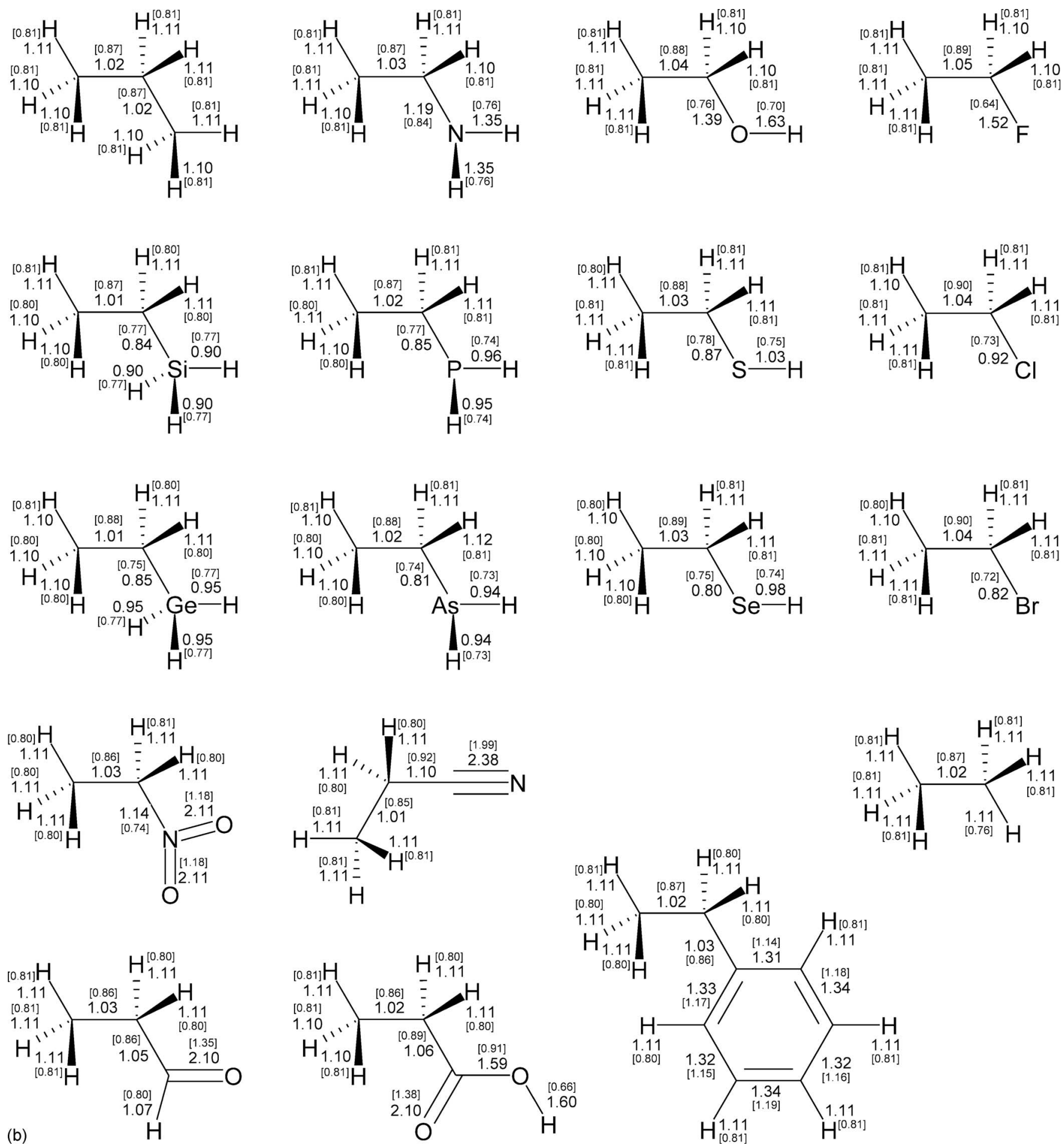

FIG. 8. (Continued).

strengthening of butadiyne bonding by 0.150 . However, interatomic regions by themselves point lack of conjugative stabilization or even destabilization in butadiyne.

These conclusions might also be derived from the series [Eqs. (20)-(22)] where stronger triple bonds of butadiyne than vinylacetylene or stronger double bonds of vinylacetylene than 1,3-butadiene indicate less partial bond equalization in the former molecule and more in the latter, thus weaker and stronger conjugations, respectively. However, the adjacent single $\mathrm{C}-\mathrm{C}$ bonds strengthen in a contrasting manner suggesting stronger conjugation in the butadiyne mol- ecule, yet this is misleading. The very presence of $\pi$ bonds affects neighboring $\sigma$ bonds by increasing their strengths. One may compare peripheral $\mathrm{C}-\mathrm{H}$ bonds (and others) in the vicinity of carbon-carbon $\sigma$ and $\pi$ bonds (Figs. 8(a), 8(b), 9(a), 9(b), and 10). Moreover the $\pi$ bonds in 1,3-butadiene and vinylacetylene molecules are involved in hyperconjugative stabilization with $\mathrm{C}-\mathrm{H}$ bonds around the single $\mathrm{C}-\mathrm{C}$ bond, which is competitive to conjugation effects on the latter.

Similar reasoning cannot follow from Mayer's bond order index, which yields unreasonable results of $\mathrm{C}_{4}$ hydrocar- 

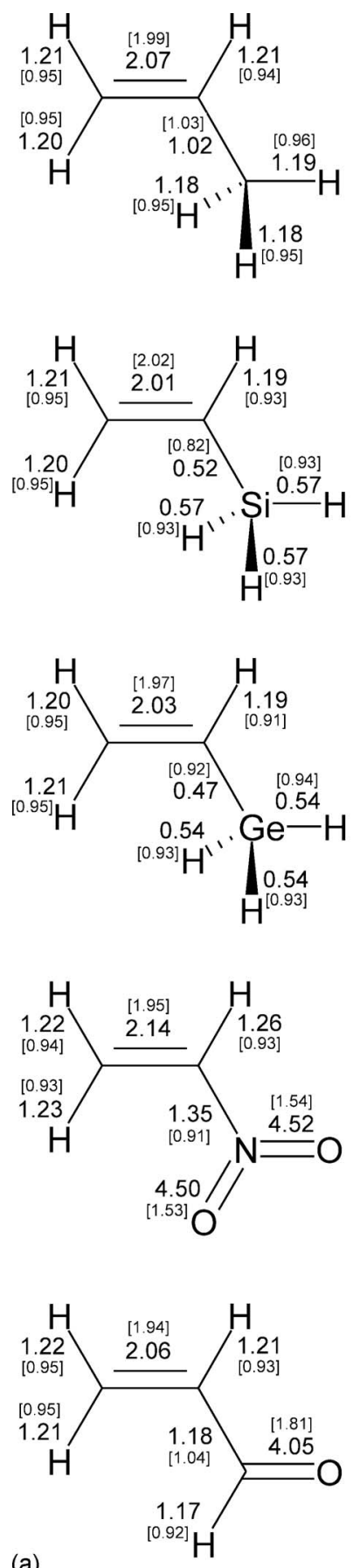

(a) FIG. 9. The electronic stress tensor rooted bond orders of bonds in $\mathrm{C}_{2} \mathrm{H}_{3} \mathrm{~A}$
As $\mathrm{H}_{2}, \mathrm{OH}, \mathrm{SH}, \mathrm{SeH}, \mathrm{F}, \mathrm{Cl}$, and $\mathrm{Br}$ ): (a) Energy density based bond order $b$
order $b_{\mu}$, overlap-weighted NAO bond order in parentheses.
bons, like the negative bond order of the triple bond of vi-
nylacetylene (Table V). Negative values are attributed to the presence of diffuse functions, ${ }^{40}$ which in contrast have no noticeable adverse effect on $b_{\varepsilon}$ and $b_{\mu}$.

\section{Orbitalwise contributions}

The MOs involved in conjugation or hyperconjugation effects were recognized by orbitalwise analysis. Table VII presents orbitalwise contributions to total bond orders of carbon-carbon bonds for selected $\mathrm{C}_{4}$ hydrocarbons. The greatest contribution to total bond order in
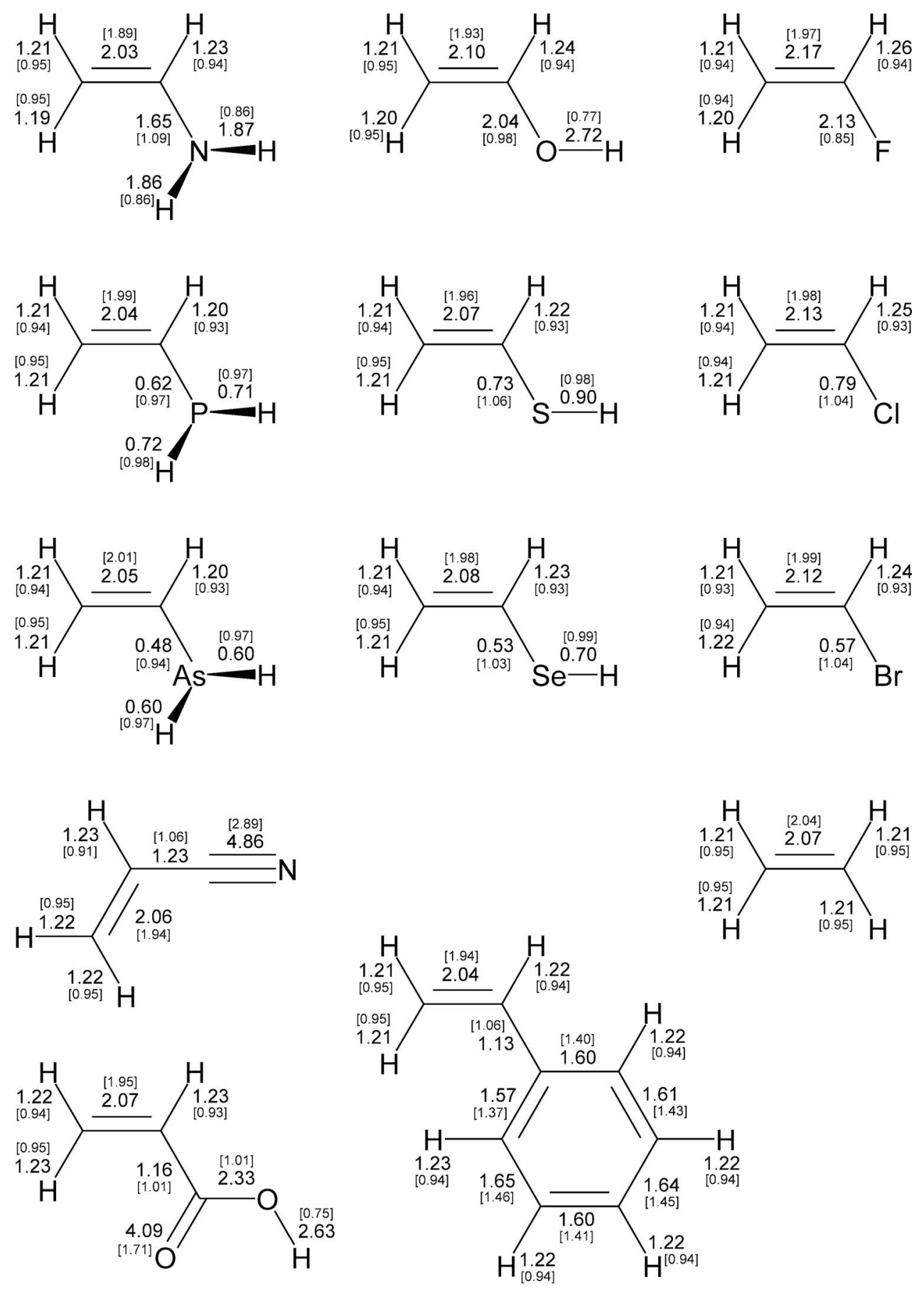

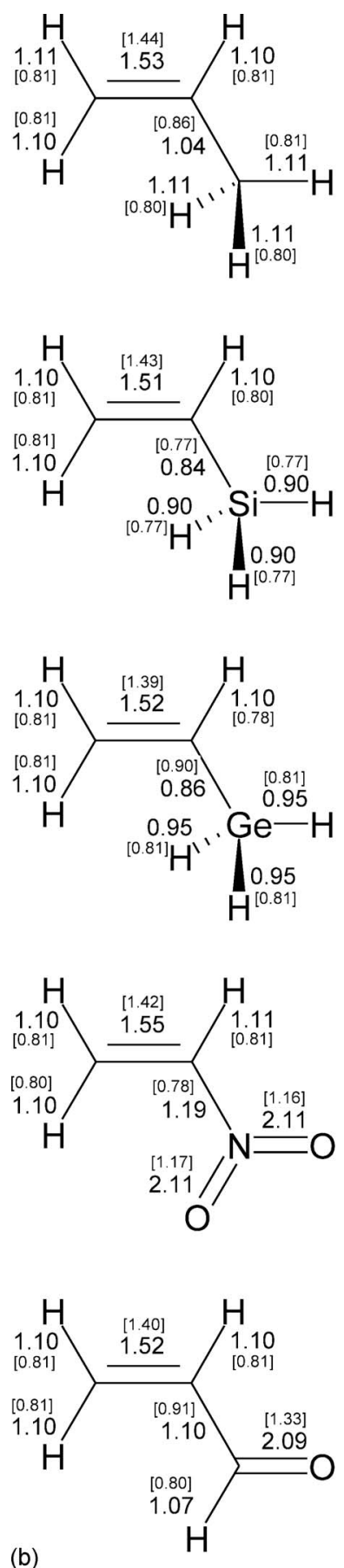
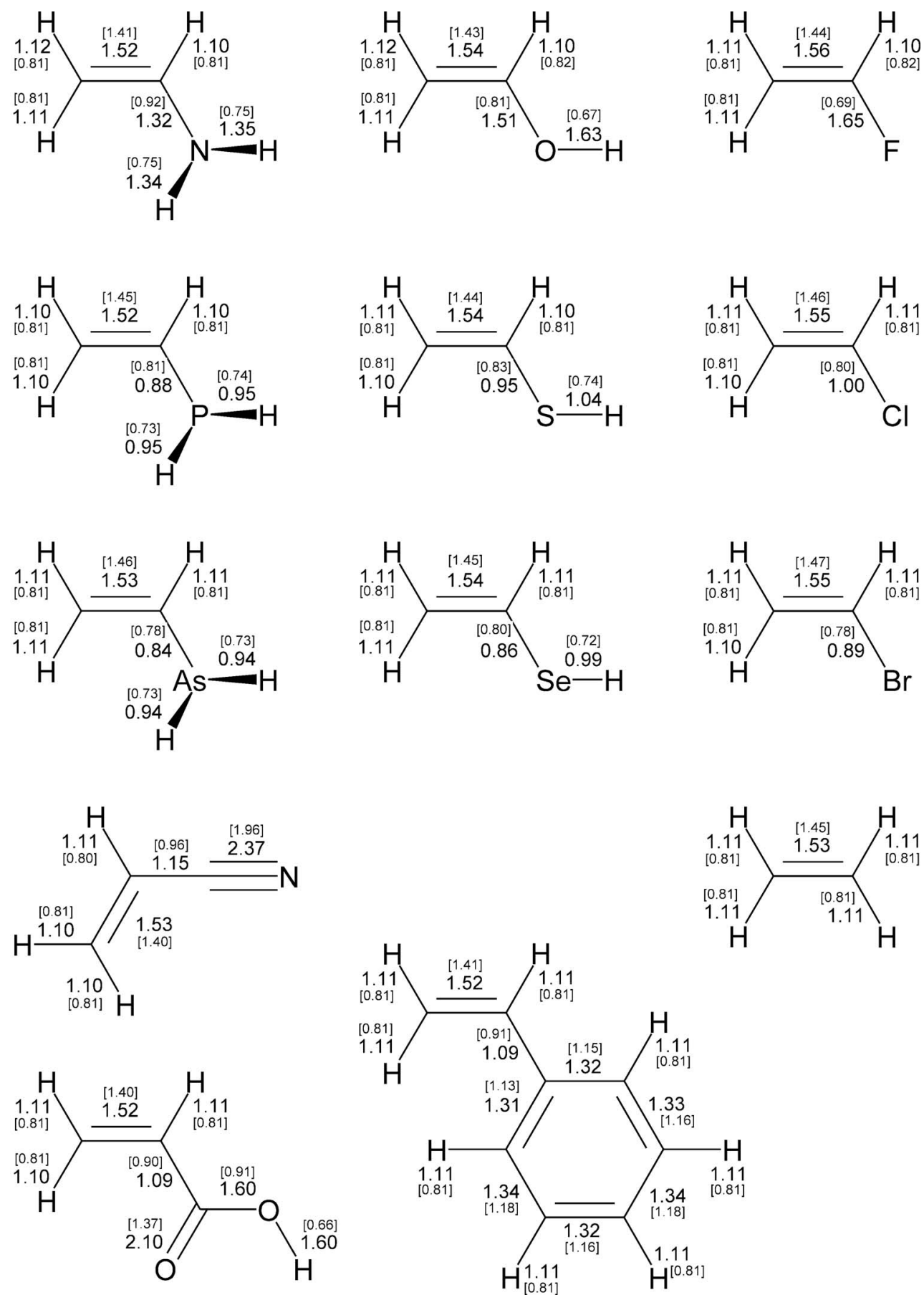

FIG. 9. (Continued) tributions from tenth, seventh, and sixth MOs, respectively. The fifth MO is the only one significantly contributing to all $\mathrm{C}$ to $\mathrm{C}$ bonds. The 12 th $\mathrm{MO}$ could also be involved in conjugation effect. However, its contribution to double bond is slightly below average, which might mean not too favorable interaction between $\pi$ orbitals (due to the greater overlap of $\pi^{*}$ orbitals).

\section{CONCLUSIONS}

The current study is an extension to electronic stress tensor representation and analysis ${ }^{8,14,15}$ of chemical interaction by means of RDFT. The stress tensors used to describe the internal forces of matter when applied to nanoscale systems, at quantum mechanical level, can characterize effects related to internal distortion of the electron density in molecules. The half of the trace over eigenvalues of the stress tensor gives local contribution to the potential energy density of electrons. The very low negative stress regions associated with atomic cores may invoke significant perturbation in the bonding region at short interatomic distances. This atomic stabilization results from low lying valence MOs having strong likeness to atomic core levels. In contrast to similar analysis presented by other researchers, ${ }^{29}$ based on topological parameters, the RDFT defines dynamical point in chemical systems at stationary state. The greater the internal quan- 

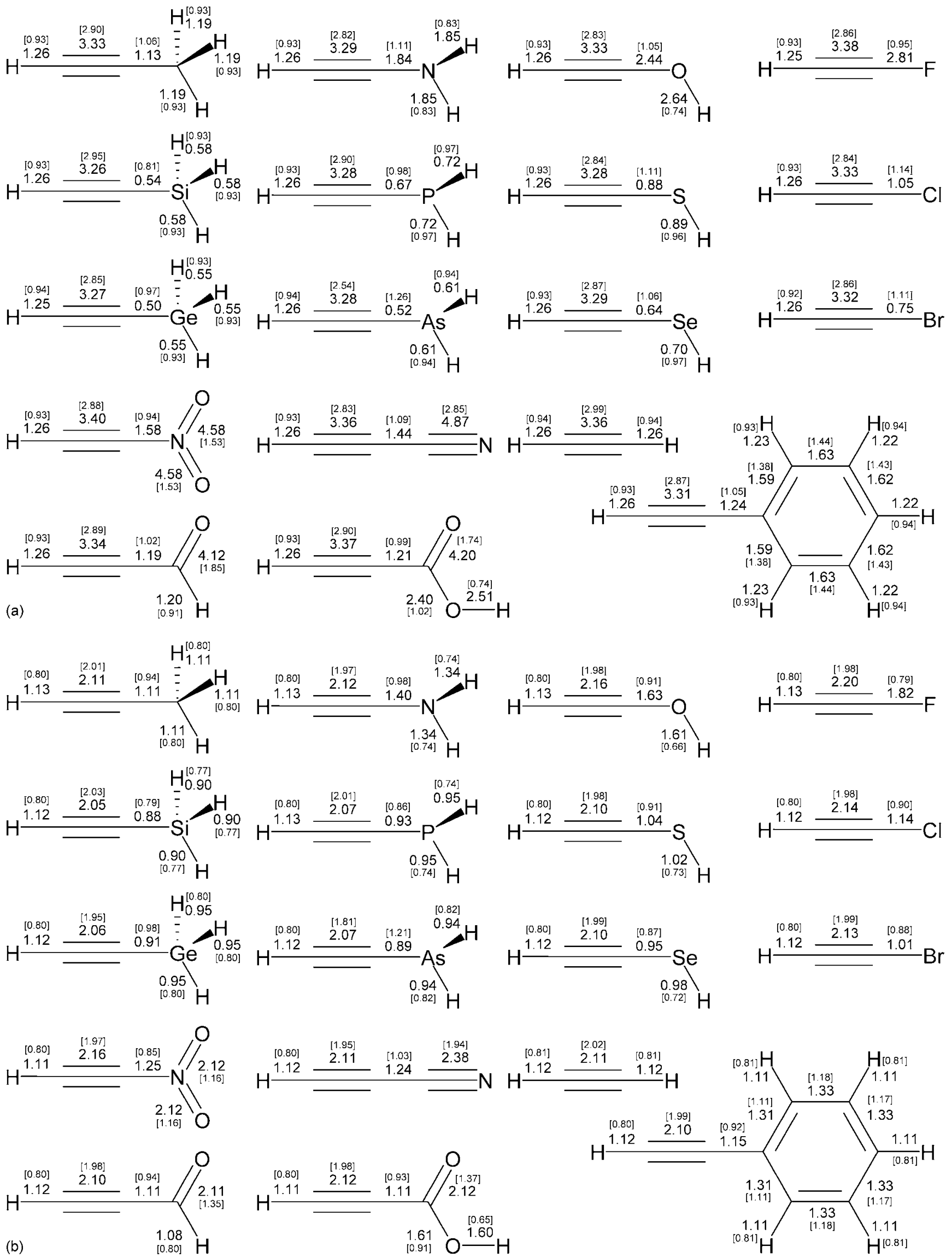

FIG. 10. The electronic stress tensor rooted bond orders of bonds in $\mathrm{C}_{2} \mathrm{HA}$ molecules $\left(\mathrm{A}\right.$ is $\mathrm{H}, \mathrm{CH}_{3}, \mathrm{CHO}, \mathrm{COOH}, \mathrm{CN} \mathrm{C}_{6} \mathrm{H}_{5}, \mathrm{SiH}_{3}, \mathrm{GeH}_{3}, \mathrm{NH}_{2}, \mathrm{NO}_{2}, \mathrm{PH}_{2}$, $\mathrm{AsH}_{2}, \mathrm{OH}, \mathrm{SH}, \mathrm{SeH}, \mathrm{F}, \mathrm{Cl}$, and $\mathrm{Br}$ ): (a) Energy density based bond order $b_{\varepsilon}$, Wiberg index in parentheses and (b) electronic chemical potential based bond order $b_{\mu}$, overlap-weighted NAO bond order in parentheses. 
TABLE VII. Orbitalwise contributions to $b_{\varepsilon}$ bond order.

\begin{tabular}{|c|c|c|c|c|c|c|c|c|c|}
\hline \multirow[b]{2}{*}{ MO } & \multicolumn{3}{|c|}{$s$-cis-1,3-butadiene } & \multicolumn{3}{|c|}{$s$-trans-1,3-butadiene } & \multicolumn{3}{|c|}{ Vinylacetylene } \\
\hline & $\mathrm{C}=\mathrm{C}$ & $\mathrm{C}-\mathrm{C}$ & $\mathrm{C}=\mathrm{C}$ & $\mathrm{C}=\mathrm{C}$ & $\mathrm{C}-\mathrm{C}$ & $\mathrm{C}=\mathrm{C}$ & $\mathrm{C}=\mathrm{C}$ & $\mathrm{C}-\mathrm{C}$ & $\mathrm{C} \equiv \mathrm{C}$ \\
\hline $1 \mathrm{st}$ & 0.000 & -0.002 & 0.000 & 0.000 & -0.003 & 0.000 & 0.001 & 0.001 & 0.000 \\
\hline $2 \mathrm{nd}$ & 0.000 & 0.003 & 0.000 & 0.000 & 0.004 & 0.000 & 0.003 & 0.000 & 0.000 \\
\hline $3 \mathrm{rd}$ & 0.002 & 0.000 & 0.002 & 0.002 & 0.000 & 0.002 & 0.000 & 0.001 & -0.009 \\
\hline 4 th & 0.002 & 0.000 & 0.002 & 0.002 & 0.000 & 0.002 & 0.000 & 0.000 & 0.023 \\
\hline 5 th & 0.232 & 0.130 & 0.232 & 0.223 & 0.147 & 0.223 & 0.347 & 0.170 & 0.252 \\
\hline 6th & 0.335 & 0.003 & 0.335 & 0.342 & 0.009 & 0.342 & 0.209 & 0.003 & 1.074 \\
\hline 7th & 0.032 & 0.219 & 0.032 & 0.027 & 0.236 & 0.027 & 0.030 & 0.335 & 0.140 \\
\hline 8th & 0.080 & 0.005 & 0.080 & 0.043 & 0.026 & 0.043 & 0.060 & 0.040 & 0.227 \\
\hline 9th & 0.093 & 0.139 & 0.093 & 0.323 & 0.050 & 0.323 & 0.110 & 0.217 & 0.415 \\
\hline 10th & 0.452 & 0.139 & 0.452 & 0.127 & 0.165 & 0.127 & 0.880 & 0.157 & 0.110 \\
\hline 11th & 0.235 & 0.001 & 0.235 & 0.551 & 0.075 & 0.551 & 0.014 & 0.187 & 0.076 \\
\hline 12 th & 0.221 & 0.004 & 0.221 & 0.069 & 0.020 & 0.069 & 0.135 & 0.117 & 0.245 \\
\hline 13th & 0.030 & 0.386 & 0.030 & 0.003 & 0.352 & 0.003 & 0.060 & 0.025 & 0.499 \\
\hline 14 th & 0.147 & 0.102 & 0.147 & 0.146 & 0.108 & 0.146 & 0.194 & 0.001 & 0.259 \\
\hline 15 th & 0.178 & 0.000 & 0.178 & 0.177 & 0.000 & 0.177 & $\ldots$ & $\ldots$ & $\ldots$ \\
\hline$\Sigma$ & 2.036 & 1.129 & 2.036 & 2.036 & 1.188 & 2.036 & 2.044 & 1.254 & 3.311 \\
\hline Average & 0.136 & 0.075 & 0.136 & 0.136 & 0.079 & 0.136 & 0.146 & 0.090 & 0.236 \\
\hline
\end{tabular}

tum forces are the steeper the electron density changes in space are. However, at the Lagrange point internal quantum forces disappear (for stationary state). This point, being an attractor for fluxes, is observed where the density builds upand the interference pattern forms. The features of this stationary point of charge density in the interatomic region make it a specific connector between two chemically bonded atoms.

Bond orders presented here refer to bond strength relative to bonds with order of 1 and are expressed in terms of electronic energy density, thus have natural ability to fulfill this role. Moreover these new indices show the redistribution of energy over the molecule and its partition between particular bonds. The electrons, recognized as electron pairs by classically oriented bond orders, bind two atoms with different strengths, depending on the electronic energy. A greater $b_{\varepsilon}$ index, which is a measure of this energy, is usually associated with higher electron density, thus (in correspondence with density or overlap based bond orders) stronger bond. The $b_{\mu}$ index reflects very well the electronegativities of bounded partners. The stress tensor rooted bond orders show a very good correlation with bond lengths. Although stress tensor rooted indices are usually higher than the corresponding NBO bond orders, it is found that the $b_{\varepsilon}$ bond order correlates with $b_{W}$ while $b_{\mu}$ is related to $b_{\mathrm{NAO}}$. The indices show small basis set dependence manifesting in differences between indices obtained in different basis sets; however, the trends within a particular basis set are preserved. These new measures allow for recognizing relative stabilities of geometrical isomers and even conformers. One can obtain reliable and informative description of interaction using molecular properties probed at the Lagrange point.

The methods based on local kinetic energy density such as $\mathrm{AIM},{ }^{29}$ electron localized function (ELF) ${ }^{60-63}$ localizedorbital locator (LOL), ${ }^{64,65}$ or local entropy measure ${ }^{66}$ analyze the electron density redistribution using $\nabla^{2} \rho(r)$. Despite the fact that it has proved to be an important analytic tool, it is difficult to understand in simple physical terms (see Bader's explanation ${ }^{29}$ ). The other related methods (temperature of nighness ${ }^{28}$ and covariance methods ${ }^{67}$ ) measure the local correlation between electrons; thus the local probability of electron pairing is given. The Pauling described the chemical bond between two atoms as a result of forces acting between them, leading to the formation of aggregate with sufficient stability. ${ }^{68}$ The RDFT studies the force exerted on electrons, as the intense variable coupled with energy, in terms of stress tensor. The methods ${ }^{28,60-67}$ allow recognizing shell structures of atoms, and so is the RDFT kinetic energy density, on a very fundamental level, separating core from valence electron regions. The local temperature of nighness ${ }^{28}$ is a measure of kinetic energy, and similarly the stress tensor gives a measure of kinetic energy density with positive contribution from compressive stress and with negative contribution from tensile stress due to negative eigenvalues of metric tensor $g^{i j}{ }^{15}$ Most of the mentioned methods need suitable reference system to define regions where electrons are especially localized. The stress tensor analysis provides such information explicitly from system wave function, using tensile stress characterizing the covalent interaction or Lagrange point-a stationary point of electron force without relating to reference systems.

\section{ACKNOWLEDGMENTS}

This work was supported by the Japanese GovernmentMonbukagakusho Scholarship through Kyoto University.

${ }^{1}$ I. Mayer, Chem. Phys. Lett. 97, 270 (1983).

${ }^{2}$ I. Mayer, Int. J. Quantum Chem. 23, 341 (1983).

${ }^{3}$ M. A. Natiello and J. A. Medrano, Chem. Phys. Lett. 105, 180 (1984).

${ }^{4}$ E. D. Glendening and F. J. Weinhold, J. Comput. Chem. 19, 610 (1998).

${ }^{5}$ I. Mayer, J. Comput. Chem. 28, 204 (2007).

${ }^{6}$ A. Sekiguchi, R. Kinjo, and M. Ichinohe, Science 305, 1755 (2004).

${ }^{7}$ M. Lein, A. Krapp, and G. J. Frenking, J. Am. Chem. Soc. 127, 6290 (2005).

${ }^{8}$ P. Szarek and A. Tachibana, J. Mol. Model. 13, 651 (2007). 
${ }^{9}$ A. Tachibana, Theor. Chem. Acc. 102, 188 (1999).

${ }^{10}$ A. Tachibana, J. Chem. Phys. 115, 3497 (2001).

${ }^{11}$ A. Tachibana, in Stress Induced Phenomena in Metallization, edited by S. P. Baker (American Institute of Physics, New York, 2002), pp. 205-211.

${ }^{12}$ A. Tachibana, in Reviews in Modern Quantum Chemistry: A Celebration of the Contributions of Robert Parr, edited by K. D. Sen (World Scientific, Singapore, 2002), Vol. 2, pp. 1327-1366.

${ }^{13}$ A. Tachibana, in Fundamental Perspectives in Quantum Chemistry: A Tribute to the Memory of Per-Olov Löwdin, edited by E. Brändas and E. Kryachko (Kluwer, Dordrecht, 2003), Vol. 2, pp. 211-239.

${ }^{14}$ A. Tachibana, Int. J. Quantum Chem. 100, 981 (2004).

${ }^{15}$ A. Tachibana, J. Mol. Model. 11, 301 (2005).

${ }^{16}$ W. A. Bingel, Z. Naturforsch. A 18A, 1249 (1963).

${ }^{17}$ W. A. Bingel, Theor. Chim. Acta 8, 54 (1967).

${ }^{18}$ T. Kato, Commun. Pure Appl. Math. 10, 151 (1957).

${ }^{19}$ R. T. Pack and W. B. Brown, J. Chem. Phys. 45, 556 (1966).

${ }^{20}$ A. Tachibana, Int. J. Quantum Chem., Quantum Chem. Symp. 21, 181 (1987).

${ }^{21}$ A. Tachibana, Int. J. Quantum Chem. 57, 423 (1996).

${ }^{22}$ A. Tachibana and R. G. Parr, Int. J. Quantum Chem. 41, 527 (1992).

${ }^{23}$ A. Tachibana, K. Nakamura, K. Sakata, and T. Morisaki, Int. J. Quantum Chem. 74, 669 (1999).

${ }^{24}$ R. F. W. Bader and P. M. Beddall, J. Chem. Phys. 56, 3320 (1972).

${ }^{25}$ L. Cohen, J. Chem. Phys. 70, 788 (1979).

${ }^{26}$ L. Cohen, J. Chem. Phys. 80, 4277 (1984).

${ }^{27}$ S. K. Ghosh, M. Berkowitz, and R. G. Parr, Proc. Natl. Acad. Sci. U.S.A. 81, 8028 (1984).

${ }^{28}$ P. W. Ayers, R. G. Parr, and A. Nagy, Int. J. Quantum Chem. 90, 309 (2002).

${ }^{29}$ R. F. W. Bader, Atoms in Molecules: A Quantum Theory (Oxford University Press, Oxford, 1990).

${ }^{30}$ A. D. Becke, Int. J. Quantum Chem. 23, 1915 (1983); T. Van Voorhis and G. E. Scuseria, Mol. Phys. 92, 601 (1997); J. Chem. Phys. 109, 400 (1998); C. Lee and R. G. Parr, Phys. Rev. A 35, 2377 (1987); A. D. Becke and M. R. Roussel, ibid. 39, 3761 (1989); R. Neumann, R. H. Nobes, and N. C. Handy, Mol. Phys. 87, 1 (1996); J. M. Tao, J. P. Perdew, V. N. Staroverov, and G. E. Scuseria, Phys. Rev. Lett. 91 , 146401 (2003).

${ }^{31}$ R. F. W. Bader, J. Chem. Phys. 73, 2871 (1980).

${ }^{32}$ A. Holas and N. H. March, Phys. Rev. A 51, 2040 (1995).

${ }^{33}$ A. Nagy and N. H. March, Mol. Phys. 91, 597 (1997).

${ }^{34}$ L. J. Bartolotti and R. G. Parr, J. Chem. Phys. 72, 1593 (1980).

${ }^{35}$ S. K. Ghosh and M. Berkowitz, J. Chem. Phys. 83, 2976 (1985).

${ }^{36}$ A. Tachibana, J. Math. Chem. 7, 95 (1991).

${ }^{37}$ M. J. Frisch, G. W. Trucks, H. B. Schlegel et al., GAUSSIAN 03, Revision C.02, Gaussian, Inc., Wallingford, CT, 2003.

${ }^{38}$ K. Doi, P. Szarek, K. Nakamura, M. Senami, and A. Tachibana, Molecular Regional DFT Program Package Version 2 (Tachbana Laboratory, Kyoto University, Kyoto, 2007).
${ }^{39}$ I. Mayer and A. Hamza, Program "APOST," Version 1.07 (Chemical Research Center, Hungarian Academy of Sciences, Budapest, 2000).

${ }^{40}$ I. Mayer, Program "BORDER," Version 1.0 (Chemical Research Center, Hungarian Academy of Sciences, Budapest, 2005).

${ }^{41}$ R. F. W. Bader, Chem.-Eur. J. 12, 2896 (2006).

${ }^{42}$ R. G. Parr, R. A. Donnelly, M. Levy, and W. E. Palke, J. Chem. Phys. 68, 3801 (1978).

${ }^{43}$ I. Mayer, Chem. Phys. Lett. 97, 270 (1983); 117, 396 (1985).

${ }^{44}$ K. B. Wiberg, Tetrahedron 24, 1083 (1968).

${ }^{45}$ F. Weinhold and J. E. Carpenter, J. Mol. Struct.: THEOCHEM 165, 189 (1988).

${ }^{46}$ A. E. Reed and P. v. R. Schleyer, Inorg. Chem. 27, 3969 (1988); J. Am. Chem. Soc. 112, 1434 (1990).

${ }^{47}$ See EPAPS Document No. E-JCPSA6-129-628834 for supplemental figures. For more information on EPAPS, see http://www.aip.org/pubservs/ epaps.html.

${ }^{48}$ T. H. Dunning, Jr. and P. J. Hay, in Modern Theoretical Chemistry, edited by H. F. Schaefer III (Plenum, New York, 1976), Vol. 3, pp. 1-28.

${ }^{49}$ D. E. Woon, Jr. and T. H. Dunning, J. Chem. Phys. 98, 1358 (1993).

${ }^{50}$ R. A. Kendall, Jr., T. H. Dunning, and R. J. Harrison, J. Chem. Phys. 96, 6796 (1992)

${ }^{51}$ J. Baker, Theor. Chim. Acta 68, 221 (1985).

${ }^{52}$ L. Pauling, J. Am. Chem. Soc. 54, 3570 (1932).

${ }^{53}$ A. L. Allred, J. Inorg. Nucl. Chem. 17, 215 (1961).

${ }^{54}$ R. P. Iczkowski and J. L. Margrave, J. Am. Chem. Soc. 83, 3547 (1961).

${ }^{55} \mathrm{~F}$. Wienhold and C. Landis, Valency and Bonding (Cambridge University Press, Cambridge, 2005).

${ }^{56}$ G. B. Kistiakowsky, J. R. Ruhoff, H. A. Smith, and W. E. Vaughan, J. Am. Chem. Soc. 58, 146 (1936).

${ }^{57}$ J. B. Conant and G. B. Kistiakowsky, Chem. Rev. (Washington, D.C.) 20, 181 (1937).

${ }^{58}$ J. B. Conn, G. B. Kistiakowsky, and E. A. Smith, J. Am. Chem. Soc. 61, 1868 (1939)

${ }^{59}$ W. Fang and D. W. Rogers, J. Org. Chem. 57, 2294 (1992).

${ }^{60}$ A. D. Becke and K. E. Edgecombe, J. Chem. Phys. 92, 5397 (1990).

${ }^{61}$ M. Kohout, Int. J. Quantum Chem. 97, 651 (2004).

${ }^{62}$ A. Savin, A. D. Becke, J. Flad, R. Nesper, H. Preuss, and H. G. Vonschnering, Angew. Chem. 30, 409 (1991).

${ }^{63}$ A. Savin, R. Nesper, S. Wengert, and T. F. Fassler, Angew. Chem. 36, 1809 (1997).

${ }^{64}$ H. L. Schmider and A. D. Becke, J. Mol. Struct.: THEOCHEM 527, 51 (2000).

${ }^{65}$ H. L. Schmider and A. D. Becke, J. Chem. Phys. 116, 3184 (2002).

${ }^{66}$ P. K. Chattaraj, E. Chamorro, and P. Fuentealba, Chem. Phys. Lett. 314, 114 (1999).

${ }^{67}$ P. W. Ayers, J. Chem. Sci. 117, 441 (2005).

${ }^{68}$ L. Pauling, The Nature of the Chemical Bond (Cornell University Press, Ithaca, 1948). 